\title{
IR-UV DOUBLE RESONANCE SPECTRUM OF ACETYLENE BELOW AND ABOVE THE PREDISSOCIATION THRESHOLD
}

\author{
MASAAKI FUJII ${ }^{a}$, SHIGEKI TANABE, YASUO OKUZAWA ${ }^{\text {b) }}$ and \\ MITSUO ITOc)
}

Department of Chemistry, Faculty of Science, Tohoku University, Sendai 980, JAPAN

a) Department of Chemistry, Graduate School of Science and Engineering, Waseda University, Ohkubo, Shinjuku-ku, Tokyo 169, JAPAN.

b) Fuji Photo Film Co. Ltd., Ashigara Research Laboratories 210, Nakanuma, Minami-Ashigara, Kanagawa 250-01, JAPAN.

c) Institute for Molecular Science, Myodaiji, Okazaki 444, JAPAN.

(Received 18 May 1993)

The $\widetilde{\mathrm{A}}^{1} \mathrm{~A}_{u} \leftarrow \tilde{\mathrm{X}}^{1} \Sigma_{g}^{+}$electronic transition of the vibrationally excited acetylene molecule was studied by IR-UV double resonance spectroscopy in gas and in a supersonic jet. The $\mathrm{C}-\mathrm{H}$ antisymmetric stretching vibration $v_{\mathrm{CH}}^{\text {ant }}$ in the $\widetilde{\mathrm{A}}$ state was clearly observed when the molecule was excited to the $v_{\mathrm{CH}}{ }^{\text {sym }}+v_{\mathrm{CH}}{ }^{\text {ant }}$ combination vibration in the $\tilde{\mathrm{X}}$ state by the IR laser. When the $v_{\mathrm{CH}}{ }^{\text {ant }}$ fundamental vibration was excited, the $\mathrm{C}-\mathrm{H}$ in-plane cis-bending vibration $v_{\text {cis }}$ (in) in the $\widetilde{\mathrm{A}}$ state was observed strongly, while $v_{\mathrm{CH}}{ }^{\text {ant }}$ almost disappeared. The difference was interpreted in terms of Fermi resonance of the $v_{\mathrm{CH}}{ }^{\text {ant }}$ fundamental vibration in $\widetilde{X}$. The predissociation threshold was newly determined to be $46,439 \sim 46,673 \mathrm{~cm}^{-1}(133.11 \pm 0.33$ $\mathrm{kcal} / \mathrm{mol}$ ). In the region above the predissociation threshold, strong vibrational mixing was found. The higher members of the progression of the trans-bending vibration starting from $v_{\mathrm{CH}}^{\text {ant }}$ were assigned. It was suggested that the nonradiative relaxation accelerated in the region above $51,744 \mathrm{~cm}^{-1}$.

KEY WORDS: IR-UV Double Resonance Spectroscopy, Multiphoton Process, Electronic, Spectrum of Acetylene, Supersonic Jet, Predissociation

\section{INTRODUCTION}

The supersonic jet technique enables us to study the vibrational and rotational structures of an excited electronic state of a molecule in detail. Most molecules in a supersonic jet are populated in the zero point level of the potential surface in the ground state, thus the spectral congestions due to hot bands are eliminated very well. As a result, the spectrum shows a simple, well-resolved rovibronic structure, which is analyzed easily. In the last decade, this technique has revealed the structures of chemically important large molecules in the excited states. However, there are still few molecules of which all the vibrational modes have been assigned in the excited 
electronic state. All the vibrational levels in the excited state do not always appear in the electronic spectrum because of the Franck-Condon factor from the zero point vibrational level. For example, usually the $\pi \pi^{*}$ electronic transition does not show a vibronic band of the $\mathrm{C}-\mathrm{H}$ vibration in the excited state. $\mathrm{C}-\mathrm{H}$ bond length does not change much in going from the ground state to the $\pi \pi^{*}$ excited state, therefore the $\mathrm{C}-\mathrm{H}$ vibrational level does not have sufficient Franck-Condon factor in the $\pi \pi^{*}$ transition.

This restriction can be removed if we can populate the molecules to a specific vibrational level other than the zero point level. The transition from the excited vibrational level will have the completely different Franck-Condon factors for the vibronic levels in the excited electronic state. Consequently, hidden vibronic levels in the excited state will be observed in the electronic spectrum of the vibrationally excited molecule. For example, the C-H vibration in the $\pi \pi^{*}$ state will be clearly observed when the molecules are populated in the $\mathrm{C}-\mathrm{H}$ vibration in the ground state. The electronic transition of the vibrationally excited molecule is important not only for the spectroscopy but also for the dynamics. The molecule in the overtone vibrational level will show an intense transition to a higher vibronic level in the excited state, where the relaxation and the photochemical reaction will be activated.

Recently, two possibilities of the vibrational excitation have been proposed. One is stimulated Raman pumping and another is, of course, excitation by an IR laser. The stimulated Raman pumping can be achieved by two lasers in the visible region, such as the second harmonics of a YAG laser and the YAG pumped dye laser. A visible dye laser is a popular light source, therefore the stimulated Raman pumping and the spectroscopy of the resultant vibrationally excited molecule have been developed well. This spectroscopy, called the stimulated Raman-UV optical double resonance, ${ }^{1}$ has enough high sensitivity and pumping efficiency to manifest the transition of a vibrationally excited vdW complex in a supersonic jet. ${ }^{2-5}$ Furthermore, the symmetry of the vibrational level can be determined by the depolarization ratio of the stimulated Raman process. ${ }^{6}$ On the other hand, IR excitation has not been well developed. At the present time, tunable pulsed IR laser light has been obtained by the frequency conversion of the dye laser light by the $\mathrm{H}_{2}$ Raman shifter or the differential wave generation by a nonlinear crystal. ${ }^{7}$ Since the conversion efficiency is only a small percentage of the source, the experiment becomes difficult. Consequently, the spectroscopy of the vibrationally excited molecule by the IR laser (the IR-UV double resonance spectroscopy) has been reported only for benzene $e^{8,9}$ and acetylene, ${ }^{10}$ where the latter was used to study the collisional relaxation in the ground state. However, this does not mean the advantage of the stimulated Raman pumping. Since the Raman pumping and the IR excitation are complementary for vibrational excitation, both pumping methods are necessary. This is specially so because the $\mathrm{C}-\mathrm{H}$ vibration has a large absorption coefficient for IR, while it does not have a large cross section for the Raman process. Therefore, the $\mathrm{C}-\mathrm{H}$ vibration in the excited state is the best target for IR-UV double resonance spectroscopy. Another target is the spectroscopy and dynamics of the higher vibrational levels in the excited state. The pumping efficiency of the nonlinear Raman process decreases for overtones in comparison with that of the IR excitation. Therefore the IR-UV double resonance will enable us to observe the 
higher vibrational levels in the excited state rather easily. A purely dynamical application of this idea has been reported in the study on the photodissociation of water and related molecules from the visible overtone vibration. ${ }^{11-19}$

In the present work, we applied the IR-UV double resonance spectroscopy to the $\widetilde{A}$ state of acetylene. The molecule was excited to the $\mathrm{C}-\mathrm{H}$ antisymmetric stretching vibration $v_{\mathrm{CH}}^{\text {ant }}$ or the combination vibration of $v_{\mathrm{CH}}{ }^{\text {ant }}$ and the $\mathrm{C}-\mathrm{H}$ symmetric stretching vibration $v_{\mathrm{CH}}^{\text {sym }}$ by the IR laser of $\sim 3 \mu \mathrm{m}$ or $\sim 1.5 \mu \mathrm{m}$, respectively. One of the purposes is the observation of the unassigned $\mathrm{C}-\mathrm{H}$ vibrations, $v_{\mathrm{CH}}$ ant, the in-plane and the out-of-plane cis-bending vibrations $v_{\text {cis }}\left(\right.$ in) and $v_{\text {cis }}($ out $)$ in the $\widetilde{A}$ state. For this purpose, we excited the molecule to a specific rovibrational level in the ground state by the IR laser, and probed the lower vibrational region in the $\widetilde{A}$ state. The acetylene molecule has high fluorescence quantum yield in the lower energy region of $\widetilde{A}$, therefore the transition was detected by the fluorescence (the IR-UV double resonance LIF spectroscopy, see Figure 1). Another purpose is related to the predissociation of acetylene in the $\widetilde{A}$ state. We have found the predissociation threshold in $\widetilde{A}$ between 46,339 and $46,673 \mathrm{~cm}^{-1}$ from the drastic decrease in the

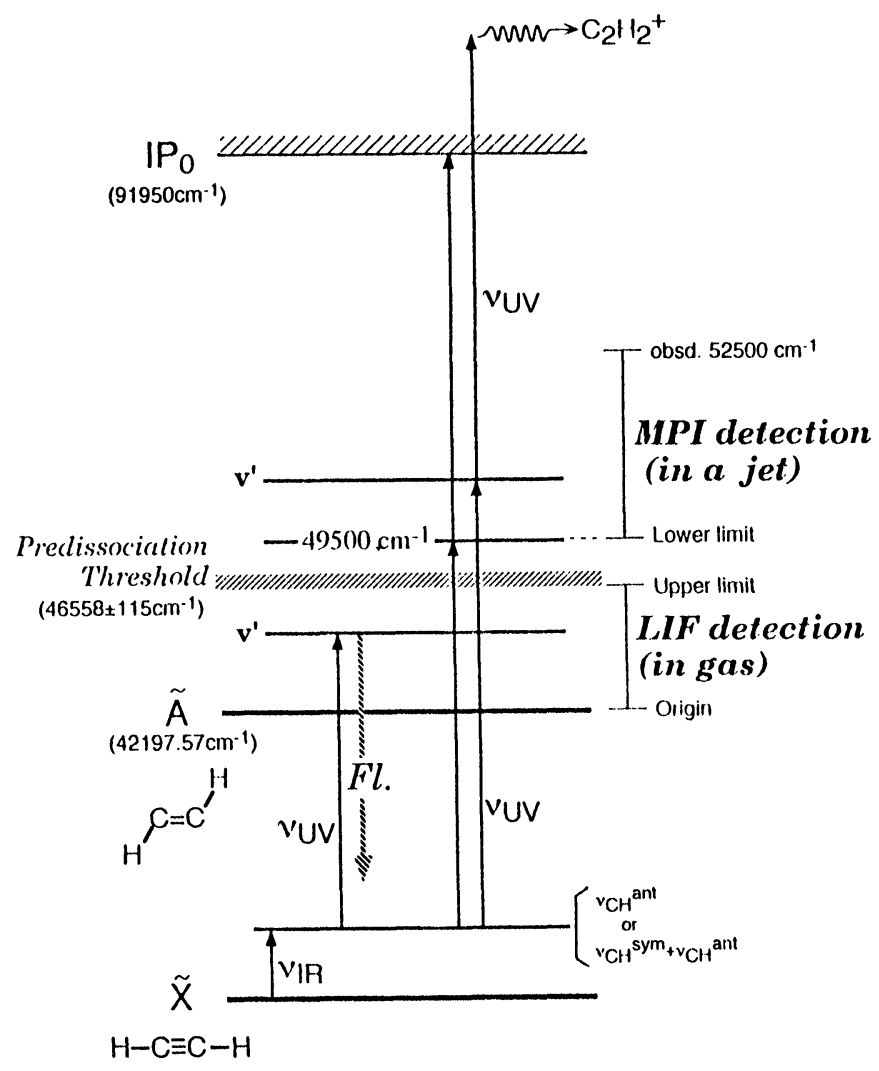

Figure 1 Schematic diagram of IR-UV double resonance spectroscopy of acetylene below and above predissociation threshold. 
fluorescence quantum yield. ${ }^{20}$ The rotational level dependence of the fluorescence quantum yield shows that its mechanism is the vibrational predissociation to $\mathrm{C}_{2} \mathrm{H}+$ $\mathrm{H}$. Based on this background, we observed the IR-UV double resonance spectrum in the region above the predissociation threshold to obtain further information on the predissociation mechanism. In the region above the predissociation threshold, the LIF detection is no longer available because of the decrease in fluorescence quantum yield. Therefore the double resonance transition was detected by the multiphoton ionization signal (see Figure 1). The molecule was excited to the $v_{\mathrm{CH}}^{\text {sym }}+v_{\mathrm{CH}}^{\text {ant }}$ combination vibration in $\bar{X}$. The vibrational motion of $v_{\mathrm{CH}}^{\mathrm{sym}}+v_{\mathrm{CH}}^{\text {ant }}$ is close to the dissociation coordinate $\mathrm{C}_{2} \mathrm{H}+\mathrm{H}$, therefore we expected the transition to a vibronic level related to the predissociation.

Very recently, Crim and co-workers have reported the near IR-UV double resonance LIF spectrum of acetylene via $3 v_{\mathrm{CH}}^{\text {ant }}$, and have assigned $v_{\text {cis }}$ (in), $v_{\text {cis }}$ (out) and $v_{\mathrm{CH}}{ }^{\text {ant }}$ in the $\widetilde{\mathrm{A}}$ state. ${ }^{21,22}$ Our assignments are consistent with theirs, and two independent observations via different vibrational levels confirm the assignments of $\mathrm{C}-\mathrm{H}$ vibrations in the $\widetilde{\mathrm{A}}$ state.

\section{EXPERIMENTAL}

The experimental apparatus for IR-UV double resonance spectroscopy is the same as that for UV-IR double resonance spectroscopy. ${ }^{23}$ Two dye lasers (Lambda Physik FL3002 and Quantel TDL 50, respectively) were pumped separately by a XeCl excimer laser (Lambda Physik LPX 100) and a Nd ${ }^{3+}:$ YAG laser (Quantel YG 581-10). The excimer laser and the YAG laser were triggered by the same pulse generator and were adjusted to give a $30 \mathrm{~ns}$ delay for the excimer laser by a digital delayedpulse generator (BNC). The UV laser light $v_{\mathrm{UV}}$ was obtained by frequency-doubling of the excimer laser pumped dye laser (dye: C500, C480 and C460) in BBO crystal, and was used to probe the $\widetilde{A}-\bar{X}$ electronic transition of acetylene. The tunable IR laser light $v_{\mathrm{IR}}$ was generated by differential mixing with the output of the YAG pumped dye laser $(\sim 640 \mathrm{~nm}, \mathrm{DCM})$ in LIO crystal (Inrad). Two kinds of mixing methods were used to generate $\nu_{\mathrm{IR}}$ depending on its wavelength region. For the generation of $\nu_{\mathrm{IR}}$ in the $1.5 \mu \mathrm{m}$ region, the dye laser light was differentially mixed with the fundamental of the YAG laser $(1.064 \mu \mathrm{m})$. The differential wave $\nu_{\mathrm{IR}}(<0.6 \mathrm{~mJ})$ was used to excite the acetylene molecule to the overtone of $\mathrm{CH}$ vibration in the $\overline{\mathrm{X}}$ state $\left(v_{\mathrm{CH}}^{\text {sym }}+v_{\mathrm{CH}}^{\text {ant }}\right)$. For the $3 \mu \mathrm{m}$ region, the dye laser light was mixed with the second harmonics of the YAG laser $(532 \mathrm{~nm})$. The differential wave $(\sim 30 \mu \mathrm{J})$ was used for the excitation of the fundamental vibration of $\mathrm{CH}$ antisymmetric stretching. Both $v_{\mathrm{IR}}$ and $v_{\mathrm{UV}}$ were coaxially introduced into a gas cell or a vacuum chamber which has a pulsed nozzle source for a supersonic jet. The acetylene molecule was excited to a specific rovibrational level in $\bar{X}$ by $v_{\mathrm{IR}}$ and was further excited to the $\widetilde{A}$ state from the vibrationally excited level by $v_{\mathrm{UV}}$. The wavelength of $v_{\mathrm{IR}}$ was fixed by measuring a photoacoustic spectrum or an infrared absorption spectrum of acetylene gas in moderate pressure ( 200 Torr) by $v_{\mathrm{IR}}$. The detail of the photoacous- 
tic spectrum of acetylene by the pulsed tunable IR laser has been described elsewhere. ${ }^{7}$ In the region below the predissociation limit $\left(42,000 \sim 47,200 \mathrm{~cm}^{-1}\right)$ in the $\widetilde{\mathrm{A}}$ state, the $\widetilde{\mathrm{A}}-\widetilde{\mathrm{X}}$ electronic transition of the vibrationally excited acetylene was detected by fluorescence from the $\widetilde{A}$ state (IR-UV double resonance LIF spectrum). The IR-UV double resonance LIF spectrum was measured in low pressure gas (10 100 $\mathrm{m}$ Torr) at room temperature. The total fluorescence was detected by a photomultiplier (Hamamatsu R-928) through an IR cut filter (Toshiba IRA-25S) at 90 degree from the lasers. In the region above the predissociation limit $\left(49,500 \sim 52,500 \mathrm{~cm}^{-1}\right)$, the transition was detected by one-photon resonant twophoton ionization signal (IR-UV double resonance MPI spectrum). IR-UV double resonance MPI spectrum was measured in a supersonic jet of pure acetylene gas $(1.5 \mathrm{~atm})$. Ions generated by $v_{\mathrm{IR}}+2 v_{\mathrm{UV}}$ were pushed by a repeller at an appropriate voltage (typically $10 \mathrm{~V} / \mathrm{cm}$ ) into a detector chamber, and were detected by a channel multiplier (Murata Ceratron). Both the fluorescence and the ion signals were amplified by an amplifier (NF for the fluorescence and Keithley for the ion) and were integrated by a digital boxcar system (EG\&G PAR 4402/4420). The integrated signal was recorded by a microcomputer (NEC).

In the present experiment, the double resonance signal was observed with the one-color signal due to $v_{\mathrm{UV}}$ only. To distinguish the double resonance signal from the one-color signal, we employed an alternative data acquisition system which was fully described elsewhere. ${ }^{23}$ Briefly, the excimer laser was operated in $20 \mathrm{~Hz}$ while the YAG laser was triggered in $10 \mathrm{~Hz}$ synchronously. Consequently, the signal due to $v_{\mathrm{IR}}+v_{\mathrm{UV}}$ and that due to $v_{\mathrm{UV}}$ only appeared alternatively. Each signal was separately integrated and was stored in a different memory array. Thus the spectrum due to $v_{\mathrm{IR}}+v_{\mathrm{UV}}$ and the spectrum due to $v_{\mathrm{UV}}$ were obtained at one time, and the double resonance signal was easily distinguished from the one-color signal by comparing both spectra. Acetylene was purchased from Teisan, and was used after passing through a solid $\mathrm{CO}_{2}$ methanol trap.

\section{RESULTS AND DISCUSSION}

\section{Notation of Vibrational Mode and Selection Rule}

It is convenient to summarize notation, symmetry and selection rule for the $\widetilde{A}-\widetilde{X}$ transition of the acetylene molecule. The geometrical structure of the acetylene molecule is linear $\left(D_{\infty \mathrm{h}}\right.$ point group) in the $\mathbb{X}^{1} \Sigma_{\mathrm{g}}{ }^{+}$state but is trans-bent $\left(\mathrm{C}_{2 \mathrm{~h}}\right)$ in the $\widetilde{\mathrm{A}}^{1} \mathrm{~A}_{\mathrm{u}}$ state. ${ }^{24-27}$ Because of this geometrical change, a long and intense progression of the trans-bent vibration appears in the $\widetilde{\mathrm{A}}-\overline{\mathrm{X}}$ transition. If the traditional notation $v_{1}, v_{2}, \ldots$ is used, the notation of vibrational mode changes in $\widetilde{X}$ and in $\widetilde{A}$ because of the geometrical change. For example, the $\mathrm{C}-\mathrm{H}$ trans bending vibration is $v_{4}{ }^{\prime \prime}$ in $\overline{\mathrm{X}}$ but is $v_{3}^{\prime}$ in $\widetilde{\mathrm{A}}$. To avoid confusion, we use the following notations: $v_{\mathrm{CH}}{ }^{\text {sym }}$ for $\mathrm{C}-\mathrm{H}$ symmetric stretching vibrational mode, $v_{\mathrm{CC}}$ for $\mathrm{C}-\mathrm{C}$ stretching, $\mathrm{V}$ for $\mathrm{C}-\mathrm{H}$ trans-bending, $v_{\mathrm{CH}}^{\text {ant }}$ for $\mathrm{C}-\mathrm{H}$ antisymmetric stretching for both states. $\mathrm{C}-\mathrm{H}$ 
cis-bending vibrational mode is denoted to be $v_{\text {cis }}$ in the $\widetilde{X}$ state. In the $\widetilde{\mathrm{A}}$ state, this mode splits into in-plane cis-bending $\nu_{\text {cis }}$ (in) and out-of-plane cis-bending $v_{\text {cis }}$ (out). The correspondence to the traditional notation is summarized in Table 1.

The symmetry of the vibrational modes is described by $\mathrm{D}_{\infty \mathrm{h}}$ and $\mathrm{C}_{2 \mathrm{~h}}$ point groups in the $\widetilde{X}$ and $\widetilde{A}$ states, respectively. The symmetry of each vibrational mode is also shown in Table 1 . When we discuss the symmetry restriction in the $\widetilde{\mathrm{A}}-\widetilde{\mathrm{X}}$ transition, the symmetry species of $D_{\infty \mathrm{h}}$ must be resolved to species in $\mathrm{C}_{2 \mathrm{~h}}$. For electronic symmetry, $\Sigma_{\mathrm{g}}{ }^{+}$for the $\widetilde{\mathrm{X}}$ state is resolved to $\mathrm{A}_{\mathrm{g}}$ in $\mathrm{C}_{2 \mathrm{~h}}$. Thus, the $\widetilde{\mathrm{A}}^{1} \mathrm{~A}_{\mathrm{u}}-\widetilde{\mathrm{X}}^{1} \Sigma_{\mathrm{g}}{ }^{+}$band is allowed transition. The symmetries for the vibrational modes in $\widetilde{X}$ are also resolved to $\mathrm{C}_{2 \mathrm{~h}}$ and are also shown in Table 1 . According to this correlation, the $v_{\mathrm{CH}}{ }^{\text {ant }}$ fundamental and the $v_{\mathrm{CH}}^{\text {sym }}+v_{\mathrm{CH}}^{\text {ant }}$ combination vibrational levels have $\sigma_{\mathrm{u}}^{+}$symmetry which is resolved to $b_{u}$ in $C_{2 h}$. Therefore, the $b_{u}$ vibrational levels in $\widetilde{A}$ are allowed in the transition from these vibrationally excited levels.

The geometrical change also affects the definition of the angular momentum. The angular momentum about the molecular axis $\mathrm{K}$ is defined to be $\mathrm{K}=| \pm \ell \pm \Lambda|$ in $\overline{\mathrm{X}}$, where $\ell$ is the vibrational angular momentum $\ell=v, v-2, v-4, \ldots, 1$ or 0 , and $\Lambda$ is the electronic angular momentum about the molecular axis. For example, both the $v_{\mathrm{CH}}^{\text {ant }}$ and $v_{\mathrm{CH}}^{\text {sym }}+v_{\mathrm{CH}}^{\text {ant }}$ vibrational levels have $\mathrm{K}=0$. On the other hand, $\mathrm{K}$ in the $\widetilde{\mathrm{A}}$ state is the rotational angular momentum about the molecular axis. Since the trans-bent structure in $\widetilde{\mathrm{A}}$ is a nearly symmetric top, the $\widetilde{\mathrm{A}}-\widetilde{\mathrm{X}}$ transition is mostly explained by the selection rule of the perpendicular transition $\Delta K= \pm 1$. Thus the $\mathrm{K}=1$ vibronic sublevel will be observed strongly in the transition from the $v_{\mathrm{CH}}^{\text {ant }}$ and $v_{\mathrm{CH}}^{\text {sym }}+v_{\mathrm{CH}}^{\text {ant }}$ vibrational levels in $\widetilde{\mathrm{X}}$.

\section{IR-UV Double Resonance Spectrum below Predissociation Threshold}

Prior to the IR-UV double resonance experiment, the frequency of the tunable IR laser $v_{\mathrm{IR}}$ must be fixed to a specific rovibrational level in the $\mathrm{X}$ state. For this purpose, the photoacoustic spectrum or the IR absorption spectrum of acetylene gas (100 200 Torr) at room temperature was measured for the fundamental vibrational band of $v_{\mathrm{CH}}{ }^{\text {ant }}$ and the combination vibrational band $v_{\mathrm{CH}}^{\text {sym }}+v_{\mathrm{CH}}^{\text {ant }}$ by $v_{\mathrm{IR}}$. The photoacoustic spectrum of acetylene by the pulsed tunable IR laser was already reported elsewhere, ${ }^{7}$ and the rovibrational structures have been well analyzed by high resolution IR spectroscopy. ${ }^{28,29}$ Briefly, the $v_{\mathrm{CH}}{ }^{\text {ant }}$ fundamental vibration shows complicated rotational structure due to Fermi resonance with $\left(v_{\mathrm{CC}}+\mathrm{V}+v_{\mathrm{cis}}\right)^{0}$ level, ${ }^{28}$ where superscript 0 shows the vibrational angular momentum $\ell$. The intensity of the Fermi pair $\left(v_{\mathrm{CC}}+\mathrm{V}+v_{\mathrm{cis}}\right)^{0}$ is almost comparable to that of $v_{\mathrm{CH}}^{\text {ant }}$; therefore, the $v_{\mathrm{CH}}^{\text {ant }}$ vibrational level contains a substantial amount of the $v_{\mathrm{CC}}+\mathrm{V}+v_{\mathrm{cis}}$ component. In contrast, the combination vibration $v_{\mathrm{CH}}^{\text {sym }}+v_{\mathrm{CH}}^{\text {ant }}$ shows a regular $\Sigma-\Sigma$ type rotational structure. ${ }^{7,28}$ It means that this combination vibrational level does not have a strong Fermi resonance, and is mostly pure vibrational level. Therefore, the IR-UV double resonance spectrum via the fundamental $v_{\mathrm{CH}}$ ant level is expected to show not only the $v_{\mathrm{CH}}{ }^{\text {ant }}$ vibration but also $v_{\text {cis }}$ vibration in the $\widetilde{\mathrm{A}}$ state. On the other hand, only the band containing the $v_{\mathrm{CH}}^{\text {ant }}$ vibration will be found in the double 


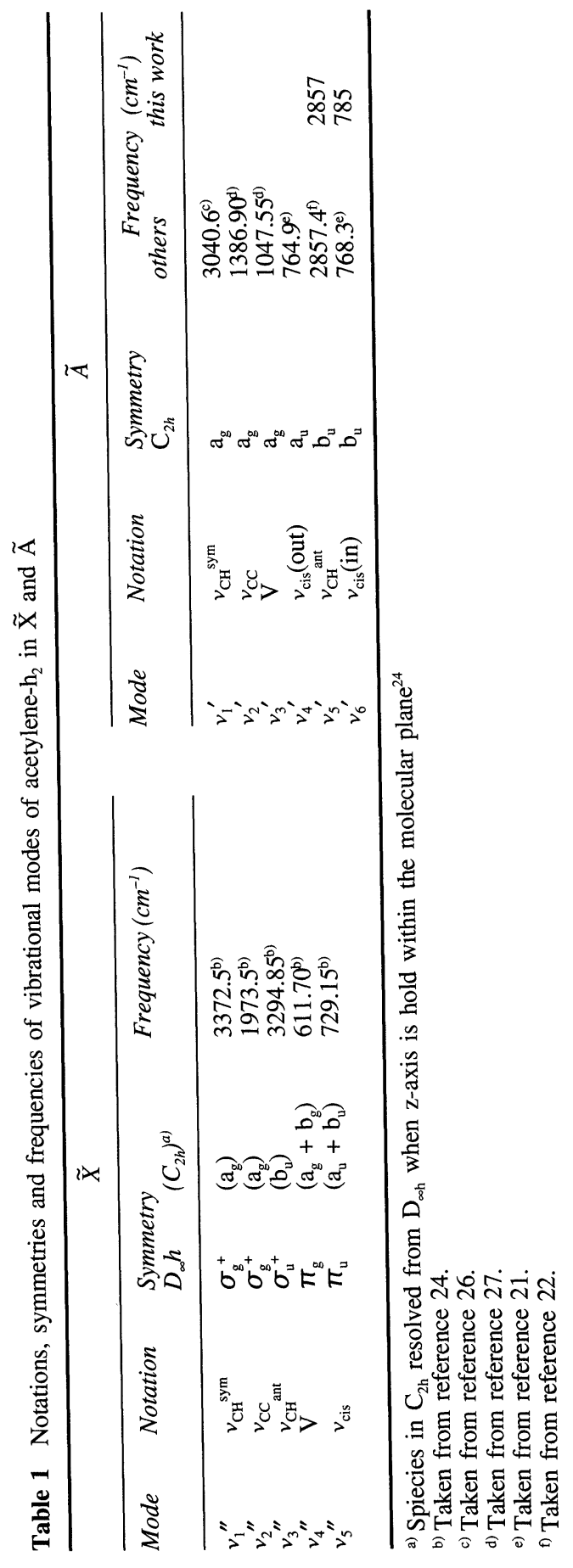


resonance spectrum when the combination vibration will be excited by $\nu_{\mathrm{IR}}$. The former is convenient to probe unknown vibrational levels in $\widetilde{A}$, and the latter is useful to confirm the assignment of $v_{\mathrm{CH}}{ }^{\text {ant }}$. By this expectation, we observed the IR-UV double resonance spectrum via the two vibrational levels.

Figure 2a shows the IR-UV double resonance LIF spectrum of acetylene gas at room temperature obtained after exciting the molecule to $\mathrm{J}^{\prime \prime}=8$ rovibrational level of the combination vibration $v_{\mathrm{CH}}^{\mathrm{sym}}+v_{\mathrm{CH}}^{\text {ant }}$ in the $\overline{\mathrm{X}}$ state. The frequency of $v_{\mathrm{IR}}$ was fixed to $\mathrm{P}(9)$ line of the combination vibrational transition. The pressure of acetylene gas was kept at $10 \mathrm{~m}$ Torr to avoid collisional relaxation. Figure $2 \mathrm{~b}$ shows the simultaneously observed spectrum due to $v_{\mathrm{UV}}$ only (see Experimental section), which corresponds to the usual $\widetilde{\mathrm{A}} \leftarrow \widetilde{\mathrm{X}}$ electronic spectrum of acetylene in the hot band region $\left(37,900-40,700 \mathrm{~cm}^{-1}\right)$. The horizontal scale was drawn by the total energy, i.e. sum of the level energy of $\mathrm{J}^{\prime \prime}=8$ of $v_{\mathrm{CH}}^{\text {sym }}+v_{\mathrm{CH}}{ }^{\text {ant }}$ and the energy of the UV laser. From the comparison between them, four band groups due to $v_{\mathrm{IR}}+v_{\mathrm{UV}}$ are found. A weak band group at $\sim 46,500 \mathrm{~cm}^{-1}$ is shown in an expanded scale (Figure $2 \mathrm{c})$. The three band groups indicated by solid lines consist of three lines, of which the spacing among them is 14 and $23 \mathrm{~cm}^{-1}$. These values match the $\mathrm{P}(8)-\mathrm{Q}(8)$ and $\mathrm{Q}(8)-\mathrm{R}(8)$ spacing in the typical rovibronic structure in $\widetilde{\mathrm{A}}-\widetilde{\mathrm{X}}$ system (14.24 and

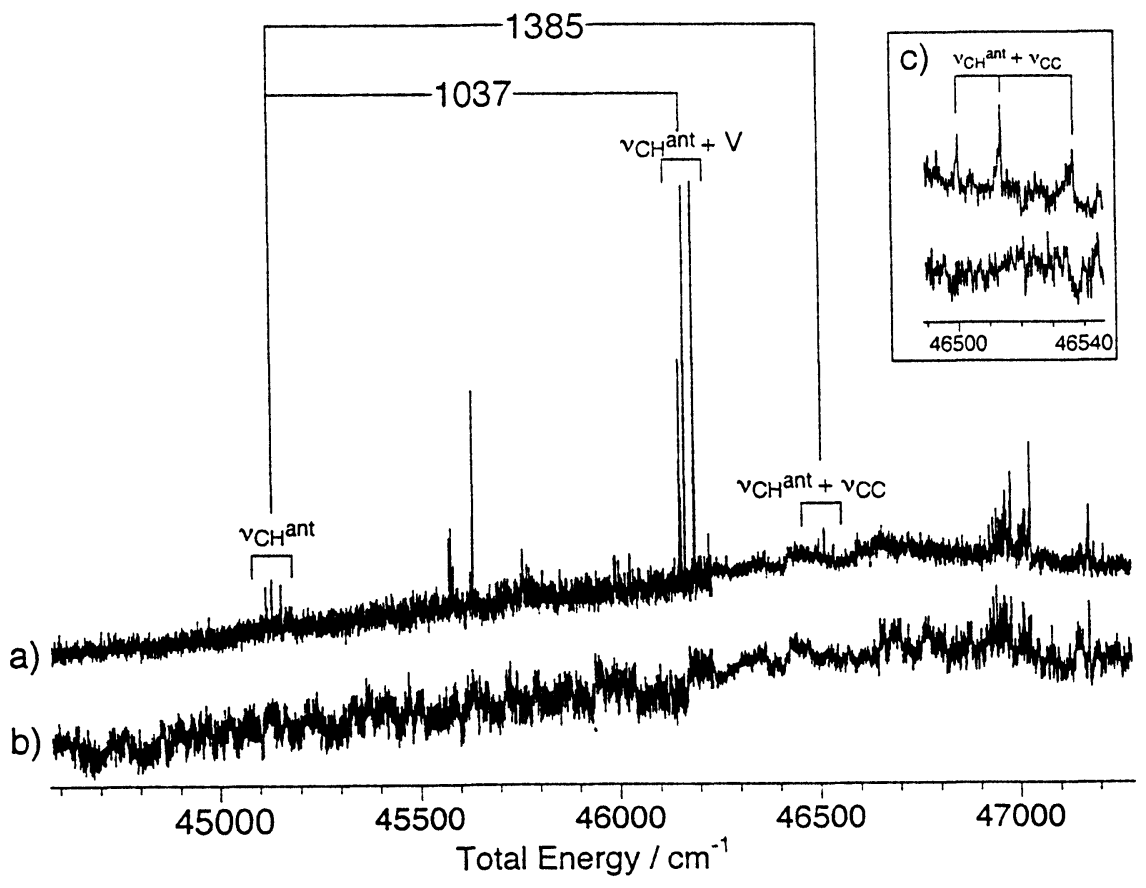

Figure 2 a) IR-UV double resonance LIF spectrum of gaseous acetylene (10 $\mathrm{m}$ Torr), b) simultaneously observed one-color LIF spectrum without $v_{\mathrm{IR}}$, and c) portions around $46,520 \mathrm{~cm}^{-1}$ drawn in an expanded scale. The spectra were obtained by exciting the molecule to $\mathrm{J}^{\prime}=8$ level in the $v_{\mathrm{CH}}^{\text {sym }}+v_{\mathrm{CH}}^{\text {ant }}$ vibration by $v_{\mathrm{IR}}$. Assignments are shown by solid lines. The horizontal scales were drawn by the total energy measured from the zero point level of $\tilde{\mathrm{X}}$. 
$23.13 \mathrm{~cm}^{-1}$ in the transition of $\left.\widetilde{\mathrm{A}} \mathrm{V}=0, \mathrm{~K}=1 \leftarrow \widetilde{\mathrm{X}} \mathrm{V}=0, \mathrm{~K}=0\right) .{ }^{27}$ Therefore the three band groups are concluded to be the transition from the combination vibrational level excited by $v_{\mathrm{IR}}$. The rest group at $\sim 45,600 \mathrm{~cm}^{-1}$ consists of four lines, and the spacing does not match the typical value shown above. Thus it is hard to conclude that this band group is the $\widetilde{A}-\widetilde{X}$ transition from the $\mathrm{J}^{\prime \prime}=8$ of vibrationally excited acetylene. This group may be due to the transition to an unknown electronic state such as cis-bent state; however, we cannot make any definite conclusion at the present time. The observed frequencies for $v_{\mathrm{UV}}$, total energies from zero rovibrational level in $\widetilde{X}$ and the excess energies from the origin of the $\widetilde{A}$ state $\left(42,197.57 \mathrm{~cm}^{-1}\right)$ are listed in Table 2.

Table 2 Positions $\left(\mathrm{cm}^{-1}\right)$ and assignments of vibronic bands observed in IR-UV double resonance LIF spectrum of acetylene obtained by exciting $\mathrm{P}(9)$ of $v_{\mathrm{CH}}^{\text {sym }}+v_{\mathrm{CH}}^{\text {ant }}$ in $\tilde{\mathrm{X}}$

\begin{tabular}{|c|c|c|c|c|c|c|}
\hline$v_{\mathrm{UV}}$ & $E_{\text {total }}^{\text {a) }}$ & $E_{\text {rovib. }}^{\mathrm{b})}$ & Branch & $\begin{array}{l}E_{\text {rovib. }}{ }^{\mathrm{c})} \\
K=1, J=1\end{array}$ & $E_{\text {vib. }}^{\text {d) }}$ & Assignment \\
\hline $\begin{array}{l}38488.4 \\
38502.8 \\
38526.2\end{array}$ & $\begin{array}{l}45128.7 \\
45143.1 \\
45166.5\end{array}$ & $\begin{array}{l}2931.1 \\
2945.5 \\
2968.9\end{array}$ & $\begin{array}{l}\mathrm{P}(8) \\
\mathrm{Q}(8) \\
\mathrm{R}(8)\end{array}$ & 2871.1 & 2857 & $v_{\mathrm{CH}}^{\text {ant }}$ \\
\hline $\begin{array}{l}38933.6 \\
38938.0 \\
38945.1 \\
38994.4\end{array}$ & $\begin{array}{l}45573.9 \\
45578.3 \\
45585.4 \\
45634.7\end{array}$ & $\begin{array}{l}3376.3 \\
3380.7 \\
3387.8 \\
3442.1\end{array}$ & & & & \\
\hline $\begin{array}{l}39524.2 \\
39537.9 \\
39561.2\end{array}$ & $\begin{array}{l}46164.5 \\
46178.2 \\
46201.5\end{array}$ & $\begin{array}{l}3966.9 \\
3980.6 \\
4003.9\end{array}$ & $\begin{array}{l}P(8) \\
Q(8) \\
R(8)\end{array}$ & 3908.1 & 3894 & $v_{\mathrm{CH}}^{\text {ant }}+\mathrm{V}$ \\
\hline $\begin{array}{l}39871.0 \\
39884.9 \\
39907.6\end{array}$ & $\begin{array}{l}46511.3 \\
46525.2 \\
46547.9\end{array}$ & $\begin{array}{l}4313.7 \\
4327.6 \\
4350.3\end{array}$ & $\begin{array}{l}\mathrm{P}(8) \\
\mathrm{Q}(8) \\
\mathrm{R}(8)\end{array}$ & 4255.6 & 4241 & $v_{\mathrm{CH}}^{\text {ant }}+v_{\mathrm{CC}}$ \\
\hline
\end{tabular}

a) Total energy from the zero rovibrational level in $\tilde{\mathrm{X}}$.

b) Rovibrational energy in $\widetilde{A}$ measured from the origin of $\widetilde{A}\left(42,197.57 \mathrm{~cm}^{-1}\right)$.

c) Rovibrational energy of $\mathrm{K}=1, \mathrm{~J}=1$ level in $\widetilde{\mathrm{A}}$ obtained from observed $\mathrm{P}$ and $\mathrm{R}$ lines.

d) Estimated vibrational energy by assuming the typical value $\left(13.1 \mathrm{~cm}^{-1}\right)$ for the rotational constant $\mathrm{A}$.

Let us discuss the vibrational assignments of three band groups due to the transition of the vibrationally excited acetylene. In our experiment, a small amount of molecules can be excited to the combination vibrational level because of the small absorption cross section. Therefore all the observed bands should be assigned to an allowed transition. As described in the last section, the level of $b_{u}$ symmetry is allowed in the transition from the $v_{\mathrm{CH}}^{\text {sym }}+v_{\mathrm{CH}}{ }^{\text {ant }}$ level $\left(\sigma_{\mathrm{u}}{ }^{+}\right.$in $\mathrm{D}_{\text {sh }}, \mathrm{b}_{\mathrm{u}}$ in $\left.\mathrm{C}_{2 \mathrm{~h}}\right)$. Therefore the lowest frequency band group at $45,143 \mathrm{~cm}^{-1}$ (for $\mathrm{Q}(8)$ ) should be the transition to the fundamental vibration in $b_{u}$ symmetry. According to the symmetry correlation in Table 1 , the vibrational mode in $b_{u}$ symmetry is the in-plane cis-bending mode $v_{\text {cis }}\left(\right.$ in) or the $\mathrm{C}-\mathrm{H}$ antisymmetric stretching mode $v_{\mathrm{CH}}$ ant. The bending vibration $v_{\text {cis }}$ (in) does not match the substantial rovibrational energy $\left(2,945.5 \mathrm{~cm}^{-1}\right)$ of this fundamental band. Therefore we conclude that this band is the transition to the fundamental vibration of $v_{\mathrm{CH}}^{\text {ant }}$ in $\widetilde{\mathrm{A}}$. The other two groups appear at 1,035 and 
$1,382 \mathrm{~cm}^{-1}$ higher energy from the $v_{\mathrm{CH}}^{\text {ant }}$ band. The differences of 1,035 and $1,382 \mathrm{~cm}^{-1}$ are close to the vibrational frequencies of the trans-bending mode $\mathrm{V}$ $\left(1,047.55 \mathrm{~cm}^{-1}\right)$ and the $\mathrm{C}=\mathrm{C}$ stretching mode $v_{\mathrm{CC}}\left(1,386.90 \mathrm{~cm}^{-1}\right)$, respectively. ${ }^{27}$ Therefore, it is concluded that the bands at $1,035 \mathrm{~cm}^{-1}$ and at $1,382 \mathrm{~cm}^{-1}$ are assigned to the $v_{\mathrm{CH}}^{\text {ant }}+\mathrm{V}$ and $v_{\mathrm{CH}}^{\text {ant }}+v_{\mathrm{CC}}$ vibration in the $\widetilde{\mathrm{A}}$ state, respectively. The strong appearance of the $v_{\mathrm{CH}}{ }^{\text {ant }}+\mathrm{V}$ vibration is consistent with the geometrical change in going from the linear $\widetilde{X}$ state to the trans-bent $\widetilde{A}$ state.

To obtain the pure vibrational frequency of $v_{\mathrm{CH}}^{\text {ant }}$ in the $\widetilde{\mathrm{A}}$ state, rotational energy must be subtracted from the observed energy of the $v_{\mathrm{CH}}{ }^{\text {ant }}$ vibration. It is best to measure rotational energy by measuring the IR-UV double resonance spectrum from the various $\mathrm{J}^{\prime \prime}$ levels in the combination vibration in $\mathrm{X}$. Unfortunately, we could not pump the molecule to the rovibrational levels other than $\mathrm{J}^{\prime \prime}=8$ because of the insufficient laser power of $\nu_{\mathrm{IR}}$. As a result, $\mathrm{J}^{\prime}=1, \mathrm{~K}=1$ level energy was obtained to be $2,871.1 \mathrm{~cm}^{-1}$ from the observed level energies of $\mathrm{P}(8)$ and $\mathrm{R}(8)$. Using the typical value of the rotational constant A $\left(13.1 \mathrm{~cm}^{-1}\right.$ for the zero vibrational level), the vibrational frequency of the $v_{\mathrm{CH}}{ }^{\text {ant }}$ is estimated to be $2,857 \mathrm{~cm}^{-1}$. Similarly, the pure vibrational energy was also estimated for the $v_{\mathrm{CH}}{ }^{\text {ant }}+\mathrm{V}$ and $v_{\mathrm{CH}}{ }^{\text {ant }}+v_{\mathrm{CC}}$. The values are also listed in Table 2 . Very recently, Tobiason et al. ${ }^{22}$ have also observed the $v_{\mathrm{CH}}^{\text {ant }}$ vibration in $\widetilde{\mathrm{A}}$ by the near IR-UV double resonance spectroscopy via $3 v_{\mathrm{CH}}{ }^{\text {ant }}$ in $\bar{X}$. From thorough rotational analysis, they have reported the frequency of $v_{\mathrm{CH}}{ }^{\text {ant }}$ to be $2,857.4 \pm 0.2 \mathrm{~cm}^{-1}$. Our value is consistent with theirs; therefore, the assignment of mode $v_{\mathrm{CH}}$ ant in $\widetilde{\mathrm{A}}$ is confirmed by observation from two different vibrational levels in $\widetilde{X}$.

The region below the predissociation threshold is also probed from the $v_{\mathrm{CH}}^{\text {ant }}$ vibrational level in $\mathrm{X}$. Figure 3a shows the IR-UV double resonance LIF spectrum of acetylene at room temperature in the total energy region from $43,300 \sim 45,300 \mathrm{~cm}^{-1}$. The spectrum was obtained after exciting the molecule to the $\mathrm{J}^{\prime \prime}=8$ level of the $v_{\mathrm{CH}}^{\text {ant }}$ fundamental vibration in the $\overline{\mathrm{X}}$ state by $v_{\mathrm{IR}}$. The collisional relaxation was negligible because of the low pressure condition ( $100 \mathrm{~m}$ Torr). Figure $3 \mathrm{~b}$ is the simultaneously observed spectrum without $\nu_{\mathrm{IR}}$, which corresponds to the hot band region of an ordinary LIF spectrum of gaseous acetylene. Clearly, two strong band groups are found at $\sim 44,000$ and at $\sim 45,000 \mathrm{~cm}^{-1}$, i.e. 1,800 and $2,800 \mathrm{~cm}^{-1}$ from the origin of the $\widetilde{A}$ state. Both band groups consist of more than three rotational lines. It is a largely complicated structure in comparison with the spectrum via the combination vibration (Figure 2). Figure $3 \mathrm{c}$ and $3 \mathrm{~d}$ show the same spectra shown in Figure $3 \mathrm{a}$ and $3 \mathrm{~b}$, respectively, but in an expanded scale around the band group at $\sim 45,000 \mathrm{~cm}^{-1}$ where the $v_{\mathrm{CH}}$ ant vibration has been assigned. The positions of $\mathrm{P}(8)$, $\mathrm{Q}(8)$ and $\mathrm{R}(8)$ of the $v_{\mathrm{CH}}{ }^{\text {ant }}$ vibration are indicated by broken lines. As can be seen in the figure, the $v_{\mathrm{CH}}^{\text {ant }}$ vibration is also observed in this spectrum; however, its intensity is very weak. No error is expected because both the double resonance spectrum via the combination vibration and the present spectrum were obtained by exciting the molecule to the same rotational level $\mathrm{J}^{\prime \prime}=8$. Instead of the weak $v_{\mathrm{CH}}^{\text {ant }}$ vibration, a new vibronic band with $\mathrm{P}(8), \mathrm{Q}(8)$ and $\mathrm{R}(8)$ rotational structure appears strongly. The rotational assignments are indicated in the figure. In addition, the spectrum shows many weak bands, which will be discussed later. 


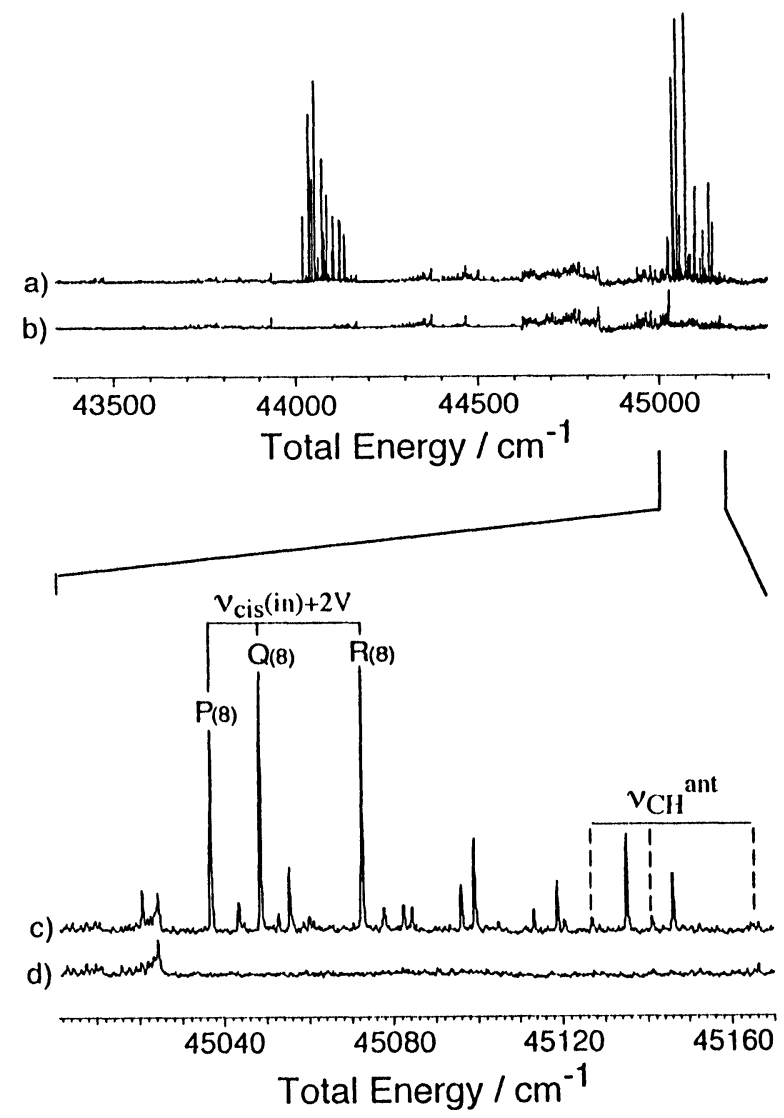

Figure 3 a) IR-UV double resonance LIF spectrum of gaseous acetylene (100 m Torr), b) simultaneously observed one-color spectrum without $v_{\mathrm{IR}}$ of acetylene. The acetylene molecule was excited to $\mathrm{J}^{\prime \prime}=8$ of the $v_{\mathrm{CH}}{ }^{\text {ant }}$ vibration by $v_{\mathrm{IR}}$. c) The IR-UV double resonance spectrum and d) the one-color spectrum around $45,000 \mathrm{~cm}^{-1}$ in an expanded scale. Assignments are shown by solid lines. The horizontal scales were drawn by the total energy measured from the zero point level of $\tilde{\mathrm{X}}$.

The strong intensity of the new vibronic band suggests that this band is also an allowed transition to the vibrational level in $b_{u}$ symmetry. Since one of the two vibrations in $b_{u}$ symmetry, $v_{\mathrm{CH}}{ }^{\text {ant }}$, has already been assigned at a higher energy region than the position of the new band, this band should be assigned to a combination vibration inducing another $b_{u}$ vibration, $v_{\text {cis }}$ (in). The strong intensity also suggests that this band contains trans-bending vibration $\mathrm{V}$ which gives larger Franck-Condon factor because of the geometrical change between $\widetilde{X}$ and $\widetilde{A}$. Another intense band group at $\sim 44,000 \mathrm{~cm}^{-1}$ is about $1,000 \mathrm{~cm}^{-1}$ below the new band; therefore, it is highly possible to assign them to be the progression of mode $\mathrm{V}$ with the false origin of $v_{\text {cis }}$ (in).

To establish the vibrational assignments, the IR-UV double resonance spectrum was measured in a wider energy region. Figure 4 shows a) the LIF spectrum due to $v_{\mathrm{IR}}+v_{\mathrm{UV}}$, and b) the LIF spectrum due to $v_{\mathrm{UV}}$ only. Both spectra are observed 


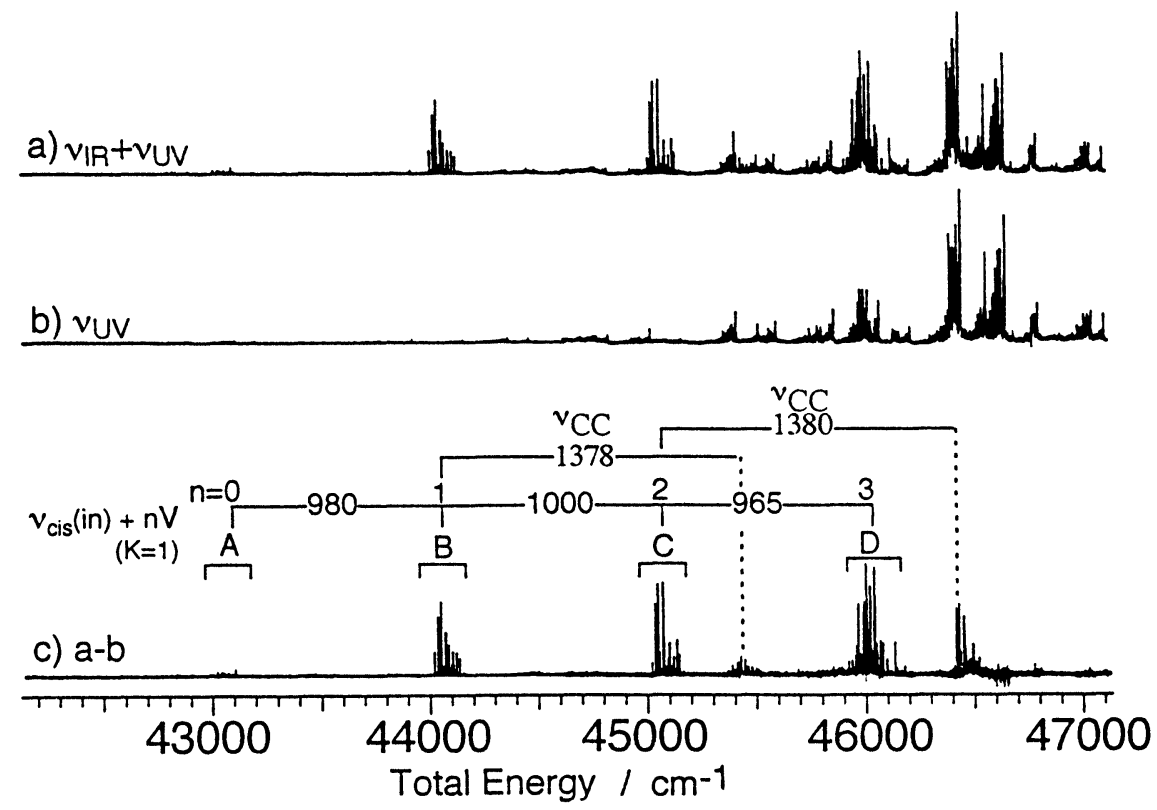

Figure 4 a) $\tilde{\mathrm{A}}-\tilde{\mathrm{X}}$ LIF spectrum of acetylene (100 m Torr) observed with $v_{\mathrm{IR}}+v_{\mathrm{Uv}}$, b) simultaneously observed one-color spectrum with $v_{\mathrm{UV}}$, and c) the spectrum obtained by subtraction $\mathrm{a}-\mathrm{b}$. The subtracted spectrum in $\mathrm{c}$ shows the band due to the IR-UV double resonance via $\mathrm{J}^{\prime \prime}=8$ of the $v_{\mathrm{CH}}^{\text {ant }}$ vibration. Assignments are indicated by solid lines. The notation of the band groups A, B, C, and D are also shown.

simultaneously by the alternative acquisition system, and $v_{\mathrm{IR}}$ was again fixed to the $\mathrm{J}^{\prime \prime}=8$ level of the $v_{\mathrm{CH}}^{\text {ant }}$ vibration in $\mathbb{X}$. Because of the observation in a wider region, intense bands due to $v_{\mathrm{Uv}}$ only overlap the double resonance bands. To obtain the spectrum only by the IR-UV double resonance, the one-color spectrum in Figure $4 \mathrm{~b}$ was subtracted from the spectrum of $v_{\mathrm{IR}}+v_{\mathrm{UV}}$ in Figure $4 \mathrm{a}$. The subtracted spectrum is shown in Figure 4c. One can see that the bands due to $v_{\mathrm{Uv}}$ only are mostly eliminated in the subtracted spectrum. In the subtracted spectrum, three intense band groups marked by B, C and D are found with $\sim 1,000 \mathrm{~cm}^{-1}$ differences in energy. The band group $B$ and $C$ are already shown in Figure 3. Furthermore, a weak band group marked by $\mathrm{A}$ is found in the region $1,000 \mathrm{~cm}^{-1}$ below the group $\mathrm{B}$. Each band group contains three intense lines which can be assigned to $\mathrm{P}(8), \mathrm{Q}(8)$ and $\mathrm{R}(8)$ from their spacing. The numbers between groups $\mathrm{A} \sim \mathrm{D}$ in the figure show the energy difference between the $\mathrm{J}=1, \mathrm{~K}=1$ level in each vibronic band calculated from $\mathrm{P}, \mathrm{Q}, \mathrm{R}$ lines. Observed band positions, excess energies from the origin, and calculated energies of $J=1$, $\mathrm{K}=1$ level energies of the intense bands are summarized in Table 3. Though the difference does not precisely match the known frequency of trans-bending mode $\mathrm{V}$ $\left(1,047.55 \mathrm{~cm}^{-1}\right)$ in $\widetilde{A}$, it is natural to assign them to the progression of vibrational mode $\mathrm{V}$. The gradual increase of the band intensity in going from A to D supports this assignment. The strong appearance of the progression suggests that the origin of the progression $\mathrm{A}$ is the fundamental vibration of $\mathrm{b}_{\mathrm{u}}$ symmetry, i.e. $v_{\mathrm{cis}}(\mathrm{in})$. The vibronic 
band A has excess energy of $797 \mathrm{~cm}^{-1}$ for $\mathrm{J}^{\prime}=1, \mathrm{~K}=1$ level, which is a reasonable frequency for the $\mathrm{C}-\mathrm{H}$ bending vibration. Therefore, we have concluded that the bands $\mathrm{A} \sim \mathrm{D}$ are the progression of $\mathrm{V}$ with the false origin of the in-plane cis-bending vibration, $v_{\text {cis }}($ in $)+\mathrm{nV}(\mathrm{n}=0 \sim 3)$. The pure vibrational energy of each member in the progression is estimated by the same procedure as that used for $v_{\mathrm{CH}}{ }^{\text {ant }}$ and is shown in Table 3. The vibrational frequency of $v_{\text {cis }}$ (in) was obtained to be $785 \mathrm{~cm}^{-1}$. The irregular spacing among the members of the progression suggests the strong perturbations in each vibronic level. Since our analysis does not consider the perturbation, the obtained value $785 \mathrm{~cm}^{-1}$ may have large uncertainty. Recently, Ulz et al. also observed the $v_{\text {cis }}$ (in) and $v_{\text {cis }}$ (out) vibrational levels in the $\widetilde{A}$ state by the near IR-UV double resonance spectroscopy via $3 v_{\mathrm{CH}}$ ant in the $\bar{X}$ state. ${ }^{21}$ The observed positions of the rovibronic bands agree with ours. Their analysis showed perturbations such as Coriolis coupling between $v_{\text {cis }}$ (in) and $v_{\text {cis }}$ (out) and centrifugal distortion, and the authors reported the unperturbed vibrational frequencies of $764.9 \pm 0.1 \mathrm{~cm}^{-1}$ and $768.3 \pm 0.2 \mathrm{~cm}^{-1}$ for $v_{\text {cis }}\left(\right.$ in) and $v_{\text {cis }}$ (out).

Table 3 Positions $\left(\mathrm{cm}^{-1}\right)$ and assignments of vibronic bands in IR-UV double resonance LIF spectrum of acetylene obtained by exciting $\mathrm{P}(9)$ of $v_{\mathrm{CH}}^{\text {ant }}$ in $\tilde{\mathrm{X}}$

\begin{tabular}{lcccccc}
\hline$v_{\mathrm{UV}}$ & $E_{\text {total }}$ & $E_{\text {rovib. }}$ b) & $\begin{array}{l}E_{\text {rovib. }}^{\text {c) }} \\
K=1, J=1\end{array}$ & Branch & $E_{\text {vib. }}$ d) & Assignment \\
\hline 39675 & 43054 & 856 & 798 & $\mathrm{P}(8)$ & 785 & $v^{\text {cis }}(\mathrm{in})$ \\
39693 & 43072 & 874 & 799 & $\mathrm{Q}(8)$ & & \\
39712 & 43091 & 893 & 799 & $\mathrm{R}(8)$ & & \\
40658 & 44037 & 1839 & 1781 & $\mathrm{P}(8)$ & 1765 & $v^{\text {cis }}(\mathrm{in})+\mathrm{V}$ \\
40672 & 44051 & 1853 & 1778 & $\mathrm{Q}(8)$ & & \\
40693 & 44072 & 1874 & 1780 & $\mathrm{R}(8)$ & & \\
41658 & 45037 & 2839 & 2781 & $\mathrm{P}(8)$ & 2765 & $v^{\text {cis }}(\mathrm{in})+2 \mathrm{~V}$ \\
41669 & 45049 & 2851 & 2775 & $\mathrm{Q}(8)$ & & \\
41693 & 45073 & 2875 & 2780 & $\mathrm{R}(8)$ & & \\
42035 & 45414 & 3216 & 3158 & $\mathrm{P}(8)$ & 3143 & $v^{\text {cis }}(\mathrm{in})+\mathrm{V}+v_{\mathrm{CC}}$ \\
42049 & 45428 & 3230 & 3155 & $\mathrm{Q}(8)$ & & \\
42069 & 45448 & 3250 & 3156 & $\mathrm{R}(8)$ & & \\
42625 & 46004 & 3806 & 3748 & $\mathrm{P}(8)$ & 3732 & $v^{\text {cis }}(\mathrm{in})+3 \mathrm{~V}$ \\
42641 & 46020 & 3822 & 3747 & $\mathrm{Q}(8)$ & & \\
42661 & 46040 & 3842 & 3748 & $\mathrm{R}(8)$ & & \\
43038 & 46417 & 4219 & 4161 & $\mathrm{P}(8)$ & 4145 & $v^{\text {cis }}(\mathrm{in})+2 \mathrm{~V}+v \mathrm{cc}$ \\
43051 & 46430 & 4232 & 4157 & $\mathrm{Q}(8)$ & & \\
43074 & 46453 & 4255 & 4161 & $\mathrm{R}(8)$ & & \\
\hline
\end{tabular}

a) Total energy from the zero rovibrational level in $\tilde{\mathrm{X}}$.

b) Rovibrational energy in $\widetilde{\mathrm{A}}$ measured from the origin of $\widetilde{\mathrm{A}}\left(42,197.57 \mathrm{~cm}^{-1}\right)$.

c) Rovibrational energy of $\mathrm{K}=1, \mathrm{~J}=1$ level in $\widetilde{\mathrm{A}}$ obtained from observed $\mathrm{P}, \mathrm{Q}$ and $\mathrm{R}$ lines.

d) Estimated vibrational energy by assuming the typical value $\left(13.1 \mathrm{~cm}^{-1}\right)$ for the rotational constant $\mathrm{A}$.

As described above, all the band groups contain many weak bands. To explain the weak bands, two factors can be taken into account. One is the appearance of the 
combination bands including $v_{\text {cis }}$ (out) which is promoted by Coriolis coupling with $v_{\text {cis }}($ in $) .{ }^{21}$ Another is the transition from a rotational level of $\left(v_{\mathrm{CC}}+\mathrm{V}+v_{\mathrm{cis}}\right)^{0}$ which is the Fermi pair of $v_{\mathrm{CH}}{ }^{\text {ant }}$ in $\mathbb{X}$. The $\left(v_{\mathrm{CC}}+\mathrm{V}+v_{\mathrm{cis}}\right)^{0}$ level lies very close to the $v_{\mathrm{CH}}{ }^{\text {ant }}$ vibrational level. Consequently, their rotational structures appear in the same region. The IR laser $v_{\mathrm{IR}}$ used in this experiment has just enough resolution to separate the rotational structure of $v_{\mathrm{CH}}{ }^{\text {ant }}$ and that of $\left(v_{\mathrm{CC}}+\mathrm{V}+v_{\mathrm{cis}}\right) .^{0}$ The center frequency of $v_{\mathrm{IR}}$ is carefully tuned to the rotational line of $v_{\mathrm{CH}}{ }^{\text {ant }}$, however a nearby rotational line of $\left(v_{\mathrm{CC}}+\mathrm{V}+v_{\mathrm{cis}}\right)^{0}$ may be weakly excited by the tail of $v_{\mathrm{IR}}$. Thus we have to consider four different transitions between two initial levels in $\bar{X}$ and two final levels in $\widetilde{A}$, of which one is the $\widetilde{A} v_{\text {cis }}$ (in) $\leftarrow \widetilde{X} v_{\mathrm{CH}^{\mathrm{C}}}^{\text {ant }}$ transition discussed above. The weak bands will be assigned to the rotational structures of the three additional transitions. Since the rotational structures of the $v_{\text {cis }}$ (in) vibration and the Coriolis interaction with $v_{\text {cis }}$ (out) have been thoroughly analyzed by Ulz et al., ${ }^{21}$ we may stop further discussion of the weak bands.

Let us discuss the difference between the IR-UV double resonance spectrum via the fundamental vibration of $v_{\mathrm{CH}}{ }^{\text {ant }}$ and that via the combination vibration of $v_{\mathrm{CH}}{ }^{\text {sym }}+$ $v_{\mathrm{CH}}^{\text {ant }}$. As described above, the double resonance spectrum via the $v_{\mathrm{CH}}^{\text {sym }}+v_{\mathrm{CH}}{ }^{\text {ant }}$ combination vibration in $\bar{X}$ shows only three vibronic bands, $v_{\mathrm{CH}}{ }^{\text {ant }}$ and its combination vibration with $\mathrm{V}$ and $v_{\mathrm{CC}}$ in $\widetilde{\mathrm{A}}$. On the other hand, when the $v_{\mathrm{CH}}^{\text {ant }}$ fundamental vibration in $\bar{X}$ is excited by $\nu_{\mathbb{I R}}$, the vibronic bands which contain $v_{\text {cis }}$ (in) appear strongly and the $v_{\mathrm{CH}}^{\text {ant }}$ vibronic band becomes very weak in the double resonance spectrum. Since both the IR-UV double resonance spectra are observed in the same region in $\widetilde{\mathrm{A}}$, the difference must be explained by the difference in the vibrational character between the $v_{\mathrm{CH}}^{\text {ant }}$ fundamental vibration and the $v_{\mathrm{CH}}^{\text {sym }}+$ $v_{\mathrm{CH}}^{\text {ant }}$ combination vibration excited by $v_{\mathrm{IR}}$. The combination vibration does not have strong Fermi resonance, therefore only the vibronic bands including single quantum of the $v_{\mathrm{CH}}{ }^{\text {ant }}$ mode has non zero value of the Franck-Condon factor in $\widetilde{\mathrm{A}}-\widetilde{\mathrm{X}}$ transition. On the other hand, the $v_{\mathrm{CH}}{ }^{\text {ant }}$ fundamental vibrational level is mixed with $\left(v_{\mathrm{CC}}+\mathrm{V}+v_{\mathrm{cis}}\right)^{0}$ by Fermi resonance in the $\mathbb{X}$ state, and has a component of $v_{\text {cis }}$ vibration. Consequently, the Franck-Condon factor from this level gives non zero value not only for the vibronic bands including $v_{\mathrm{CH}}{ }^{\text {ant }}$ but also for the vibronic bands including $v_{\text {cis }}(\mathrm{in})$. Therefore, the difference between both spectra is one of the evidences for the strong Fermi resonance of the fundamental $v_{\mathrm{CH}}{ }^{\text {ant }}$ vibration and for no vibrational mixing of the $v_{\mathrm{CH}}^{\mathrm{sym}}+v_{\mathrm{CH}}^{\text {ant }}$ combination vibration in the $\overline{\mathrm{X}}$ state.

The IR-UV double resonance LIF spectrum gives us the information on predissociation. In our previous work, ${ }^{20}$ the absorption spectrum and the fluorescence excitation spectrum of gaseous acetylene were compared. From the sudden and drastic decrease in the fluorescence quantum yield, the predissociation threshold to $\mathrm{C}_{2} \mathrm{H}+$ $\mathrm{H}$ in $\widetilde{\mathrm{A}}$ was obtained to be $46,339 \sim 46,673 \mathrm{~cm}^{-1}$. The same idea can be applied to the spectrum obtained by the IR-UV double resonance LIF spectroscopy, and the predissociation threshold can be measured by the present spectrum. In the double resonance spectrum via the fundamental vibration, the highest energy band is $v_{\text {cis }}($ in $)+2 \mathrm{~V}+v_{\mathrm{CC}}$, of which the absolute vibronic energy is $46,343 \mathrm{~cm}^{-1}$. In the double resonance LIF spectrum via the combination vibration, the highest vibronic band in energy is the $v_{\mathrm{CH}}^{\text {ant }}+v_{\mathrm{CC}}$ vibronic band. This band has an absolute vibronic 
energy of $46,439 \mathrm{~cm}^{-1}$. This energy exceeds the previously obtained lower limit $46,339 \mathrm{~cm}^{-1}$ and is larger than the vibronic energy of $v_{\text {cis }}($ in $)+2 \mathrm{~V}+v_{\mathrm{CC}}$ observed via $v_{\mathrm{CH}}{ }^{\text {ant }}$ in $\widetilde{X}$. Therefore the predissociation threshold is newly determined to be $46,439 \sim 46,673 \mathrm{~cm}^{-1}(133.11 \pm 0.33 \mathrm{kcal} / \mathrm{mol})$.

\section{IR-UV Double Resonance Spectrum above Predissociation Threshold}

In the region above the predissociation threshold $\left(46,439 \sim 46,673 \mathrm{~cm}^{-1}\right)$, the IR-UV double resonance LIF spectroscopy is no longer useful because of low fluorescence quantum yield. On the other hand, the multiphoton ionization spectroscopy becomes available to observe the IR-UV double resonance transition (IR-UV double resonance MPI spectroscopy). The lower limit in energy of the MPI detection is determined by the ionization potential of acetylene $\left(91,950 \mathrm{~cm}^{-1}\right)$. When the IR laser $v_{\mathrm{IR}}$ is fixed to the $v_{\mathrm{CH}}^{\text {sym }}+v_{\mathrm{CH}}^{\text {ant }}$ combination vibrational level $\left(6,556.5 \mathrm{~cm}^{-1}\right)$, the lower limit of the detectable region is about $49,500 \mathrm{~cm}^{-1}$, where $2 v_{\mathrm{UV}}+v_{\mathrm{IR}}$ exceeds the ionization potential. Thus the region between the predissociation threshold $\left(\sim 46,500 \mathrm{~cm}^{-1}\right)$ and the lower limit of the MPI detection $\left(49,500 \mathrm{~cm}^{-1}\right)$ still remains unrevealed. Except for this limitation, the IR-UV double resonance spectroscopy with the MPI detection is an ideal combination. The high sensitivity of MPI enables us to overcome the smaller pumping efficiency to higher overtone, and allows us to observe the weak IR-UV double resonance signal in a supersonic jet.

Figure 5a shows IR-UV double resonance MPI spectrum of jet-cooled acetylene for the $v_{\mathrm{IR}}+v_{\mathrm{UV}}$ energy region of $49,500 \mathrm{~cm}^{-1} \sim 52,500 \mathrm{~cm}^{-1}$, which is above the predissociation threshold. This spectrum was obtained by exciting the molecule to the $\mathrm{J}=2$ level of $v_{\mathrm{CH}}^{\text {sym }}+v_{\mathrm{CH}}^{\text {ant }}$ in $\bar{X}$ by $v_{\mathrm{IR}}$. The upper trace in the figure shows the spectrum in the presence of $\nu_{\mathrm{IR}}$, and the lower trace in the absence of $\nu_{\mathrm{IR}}$. Both spectra were measured simultaneously by the alternative data acquisition system. New bands due to $\nu_{\mathrm{IR}}+v_{\mathrm{UV}}$ were found at the positions indicated by A, $\mathrm{A}^{\prime}, \mathrm{B}$ and $\mathrm{C}$ in the figure. The observed positions of these bands are listed in Table 4. These bands are shown in Figure 5b in an expanded scale. As can be seen in Figure 5b, the observed band groups can be classified into the sharp band system A, B and C (FWHM $<1 \mathrm{~cm}^{-1}$ ) and the broad system $\mathrm{A}^{\prime}\left(\mathrm{FWHM} \sim 10 \mathrm{~cm}^{-1}\right)$. The difference of the band width suggests a difference in electronic origin or a difference in relaxation rate between the sharp systems and the broad system. In this energy region, three electronic states are predicted by $a b$ initio calculation ${ }^{30}$ in addition to the $\widetilde{\mathrm{A}}$ state; therefore, the electronic state of each band system must be assigned first.

The electronic state can be specified by the transition type. The predicted electronic states are ${ }^{1} \mathrm{~B}_{2}$ (cis-bent structure), ${ }^{1} \mathrm{~B}_{u}$ (trans) and ${ }^{1} \mathrm{~A}_{2}$ (cis) in addition to the $\widetilde{\mathrm{A}}^{1} \mathrm{~A}_{\mathrm{u}}$ state. From the $\overline{\mathrm{X}}^{1} \Sigma_{\mathrm{g}}{ }^{+}$state, the ${ }^{1} \mathrm{~A}_{2}$ (cis) state is forbidden and can be excluded. The transitions to the ${ }^{1} B_{2}$ state and to the ${ }^{1} B_{u}$ are parallel transitions. Only the perpendicular transition is the $\widetilde{\mathrm{A}}-\overline{\mathrm{X}}$ transition. To analyze the transition type, each band system is observed from various $\mathrm{J}$ levels in the $v_{\mathrm{CH}}^{\text {sym }}+v_{\mathrm{CH}}^{\text {ant }}$ vibrational level. Figure 6 shows the IR-UV double resonance MPI spectra for the region of the band group $\mathrm{C}$ obtained by exciting the molecule to the $\mathbf{J}=0,1$, and 2 levels of $v_{\mathrm{CH}}^{\text {sym }}$ $+v_{\mathrm{CH}}^{\text {ant }}$ by $v_{\mathrm{IR}}$. Four bands appear in the spectrum from the $\mathbf{J}=0$ level. In the 


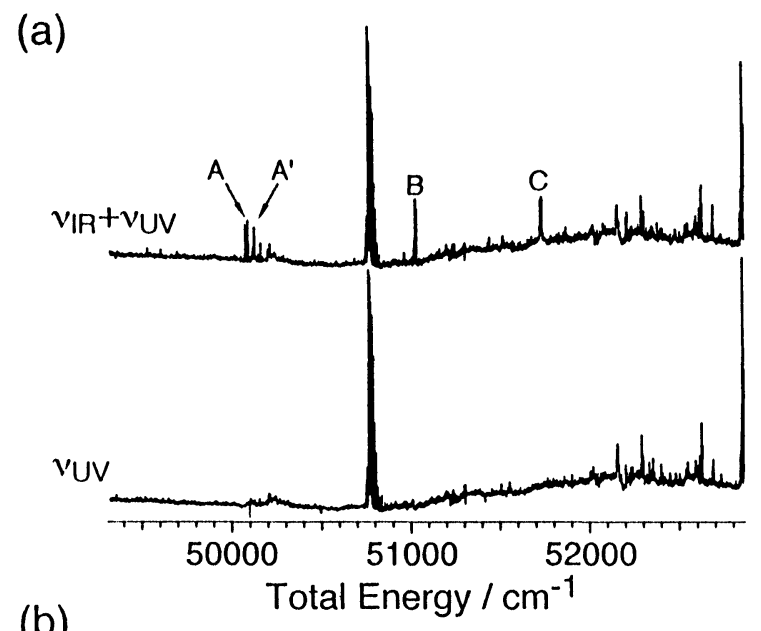

(b)

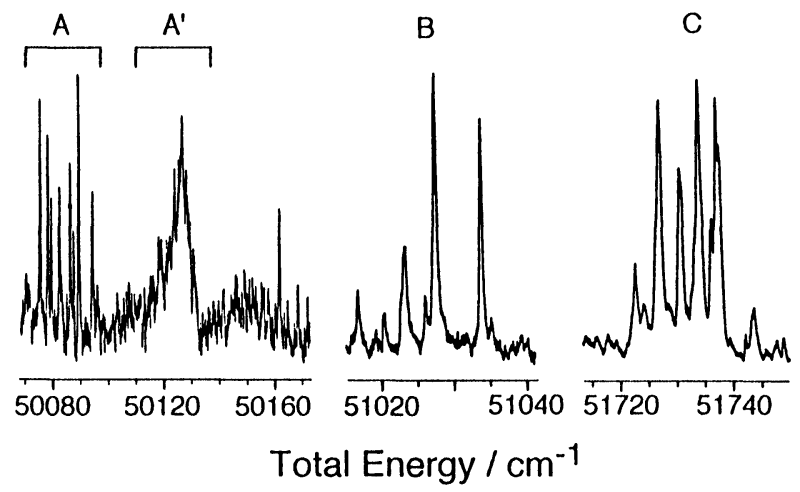

Figure 5 a) IR-UV double resonance MPI spectrum of acetylene in a supersonic jet (upper trace) and simultaneously observed MPI spectrum without $v_{\mathrm{IR}}$ (lower). $v_{\mathrm{IR}}$ was fixed to $\mathrm{J}^{\prime \prime}=2$ level in the $v_{\mathrm{CH}}^{\text {sym }}$ $+v_{\mathrm{CH}}{ }^{\text {ant }}$ combination vibration in $\widetilde{\mathrm{X}}$. The band groups due to the transition from the combination vibration are indicated by letters A, A', B, and C. b) IR-UV double resonance MPI spectrum around each band group in an expanded scale.

spectrum from $\mathbf{J}=1$, the set of the four bands observed in the spectrum from $\mathbf{J}=0$ is repeated twice with slightly different positions. The set of the four bands is indicated by a broken line. In the spectrum from $J=2$, three sets appear with slightly different positions. This feature can be interpreted to mean that 1) band group $\mathrm{C}$ consists of at least four vibronic bands with almost the same rotational constant and 2) the four vibronic bands are perpendicular transitions with the selection rule of $\Delta \mathrm{K}= \pm 1$. From the $v_{\mathrm{CH}}^{\text {sym }}+v_{\mathrm{CH}}^{\text {ant }}$ combination vibration $(\mathrm{K}=0)$ in $\mathrm{X}$, only the transition to the $K=1$ sublevel of the vibronic level in $\widetilde{A}$ is possible. The $K=1$ vibronic sublevel has rotational levels of $J \geq 1$, thus only $R(0)$ lines appear for each vibronic level when $\mathrm{J}=0$ of the combination vibration was excited by $\nu_{\mathrm{IR}}$. Thus the appearance of four $\mathrm{R}(0)$ means the existence of four vibronic levels. When the molecule is excited to $\mathrm{J}=1$ of $v_{\mathrm{CH}}^{\text {sym }}+v_{\mathrm{CH}}^{\text {ant }}$ by $\nu_{\mathrm{IR}}, \mathrm{Q}(1)$ and $\mathrm{R}(1)$ appear for 
Table 4 Positions and assignments of vibronic bands above predissociation threshold observed in IR-UV double resonance MPI spectrum of acetylene obtained by exciting $\mathrm{R}(1)$ of $v_{\mathrm{CH}}^{\mathrm{sym}}+v_{\mathrm{CH}}^{\text {ant }}$ in $\widetilde{\mathrm{X}}$

\begin{tabular}{|c|c|c|c|c|c|c|}
\hline Group $^{\text {a) }}$ & $v_{\mathrm{UV}}$ & $E_{\text {total }}^{\mathrm{b})}$ & $E_{\text {rovib }}{ }^{\mathrm{c})}$ & Branch $^{\text {d) }}$ & $E_{\mathrm{vib}}^{\mathrm{e})}$ & Assignment \\
\hline A & 43512.1 & 50075.7 & 7878.1 & $\mathrm{P}(2)$ & 7864 & $v_{\mathrm{CH}}^{\text {ant }}+5 \mathrm{~V}$ \\
\hline A & 43514.8 & 50078.4 & 7880.8 & $\mathrm{P}(2)^{\prime}$ & 7867 & \\
\hline A & 43516.1 & 50079.7 & 7882.1 & $\mathrm{Q}(2)$ & 7864 & $v_{\mathrm{CH}}^{\text {ant }}+5 \mathrm{~V}$ \\
\hline A & 43519.0 & 50082.6 & 7885.0 & $\mathrm{Q}(2)^{\prime}$ & 7867 & \\
\hline A & 43519.6 & 50083.2 & 7885.6 & $\mathrm{P}(2)^{\prime \prime}$ & 7871 & \\
\hline A & 43522.9 & 50086.5 & 7888.9 & $\mathrm{R}(2)$ & 7864 & $v_{\mathrm{CH}}^{\text {ant }}+5 \mathrm{~V}$ \\
\hline A & 43524.1 & 50087.7 & 7890.1 & $\mathrm{Q}(2)^{\prime \prime}$ & 7872 & \\
\hline A & 43525.9 & 50089.5 & 7891.9 & $\mathrm{R}(2)^{\prime}$ & 7867 & \\
\hline A & 43531.0 & 50094.6 & 7897.0 & $\mathrm{R}(2)^{\prime \prime}$ & 7872 & \\
\hline $\mathrm{A}^{\prime}$ & 43563.3 & \multicolumn{5}{|c|}{ broad band due to two-photon resonant autoionization state } \\
\hline B & 44453.3 & 51016.9 & 8819.3 & $\mathrm{P}(2)^{\prime}$ & 8805 & \\
\hline B & 44456.9 & 51020.5 & 8822.9 & $\mathrm{Q}(2)^{\prime}$ & 8804 & \\
\hline B & 44459.8 & 51023.4 & 8825.8 & $\mathrm{P}(2)$ & 8812 & $v_{\mathrm{CH}}^{\text {ant }}+6 \mathrm{~V}$ \\
\hline B & 44462.5 & 51026.1 & 8828.5 & $\mathrm{R}(2)^{\prime}$ & 8804 & \\
\hline B & 44463.8 & 51027.4 & 8829.8 & $Q(2)$ & 8812 & $v_{\mathrm{CH}}^{\text {ant }}+6 \mathrm{~V}$ \\
\hline B & 44470.1 & 51033.7 & 8836.1 & $\mathrm{R}(2)$ & 8811 & $v_{\mathrm{CH}}^{\text {ant }}+6 \mathrm{~V}$ \\
\hline $\mathrm{C}$ & 45159.0 & 51722.6 & 9525.0 & $\mathrm{P}(2)$ & 9511 & $v_{\mathrm{CH}}^{\text {ant }}+7 \mathrm{~V}(?)$ \\
\hline $\mathrm{C}$ & 45160.4 & 51724.0 & 9526.4 & $\mathrm{P}(2)^{\prime}$ & 9512 & \\
\hline C & 45163.1 & 51726.7 & 9529.1 & $\mathrm{Q}(2)$ & 9511 & $v_{\mathrm{CH}}^{\text {ant }}+7 \mathrm{~V}(?)$ \\
\hline C & 45165.0 & 51728.6 & 9531.0 & $\mathrm{Q}(2)^{\prime}$ & 9513 & \\
\hline C & 45166.7 & 51730.3 & 9532.7 & $\mathrm{Q}(2)^{\prime \prime}$ & 9514 & \\
\hline C & 45170.1 & 51733.7 & 9536.1 & $\mathrm{R}(2)$ & 9510 & $v_{\mathrm{CH}}{ }^{\text {ant }}+7 \mathrm{~V}(?)$ \\
\hline $\mathrm{C}$ & 45172.4 & 51736.0 & 9538.4 & $\mathrm{R}(2)^{\prime}$ & 9513 & \\
\hline $\mathrm{C}$ & 45173.2 & 51736.8 & 9539.2 & $\mathrm{Q}(2)^{\prime \prime \prime}$ & 9521 & \\
\hline $\mathrm{C}$ & 45173.8 & 51737.4 & 9539.8 & $\mathrm{R}(2)^{\prime \prime}$ & 9514 & \\
\hline $\mathrm{C}$ & 45179.9 & 51743.5 & 9545.9 & $\mathrm{R}(2)^{\prime \prime \prime}$ & 9521 & \\
\hline
\end{tabular}

a) Notations of the band groups shown in Figure 5a.

b) Total energies from the zero rovibrational level in $\mathrm{X}$.

c) Rovibrational energies in A measured from the origin of $\tilde{A}\left(42,197.57 \mathrm{~cm}^{-1}\right)$.

d) Assignments of rotational branches $\mathrm{P}, \mathrm{Q}$, and $\mathrm{R}$. The branches belong to the same vibronic band indicated by the same numbers of dashes, such as $\mathrm{P}(2)^{\prime \prime}, \mathrm{Q}(2)^{\prime \prime}$ and $\mathrm{R}(2)^{\prime \prime}$. The branches without dash belong to the strongest vibronic band.

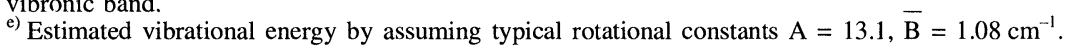

every vibronic level in $\widetilde{A}$. If the four vibronic levels have almost the same rotational constant, four $\mathrm{Q}(1)$ lines and four $\mathrm{R}(1)$ lines form two sets of bands with similar band spacing. From $J=2$, each vibronic level shows $P(2), Q(2)$ and $R(2)$ lines, and three sets of bands appear. The parallel transition cannot explain the observed spectra because of its selection rule $\Delta K=0$. Therefore these bands in group $C$ are concluded to be vibronic bands of the $\widetilde{A}$ state. Similarly, the band groups A and $\mathrm{B}$ are also concluded to be the vibronic bands of the $\widetilde{\mathrm{A}}$ state from the analysis of the transition type. It is also concluded that the band groups $\mathrm{A}, \mathrm{B}$, and $\mathrm{C}$ consist of several vibronic bands, of which the assignments are discussed later.

Figure 7 shows IR-UV double resonance MPI spectra for the region of bands A and $\mathrm{A}^{\prime}$ obtained after exciting the molecule to the $\left(v_{\mathrm{CH}}^{\text {sym }}+v_{\mathrm{CH}}{ }^{\text {ant }}\right) \mathrm{J}=0,2$, and 4 levels by $v_{\mathrm{IR}}$. The sharp band system $\mathrm{A}$ is also the cluster of the vibronic bands of 


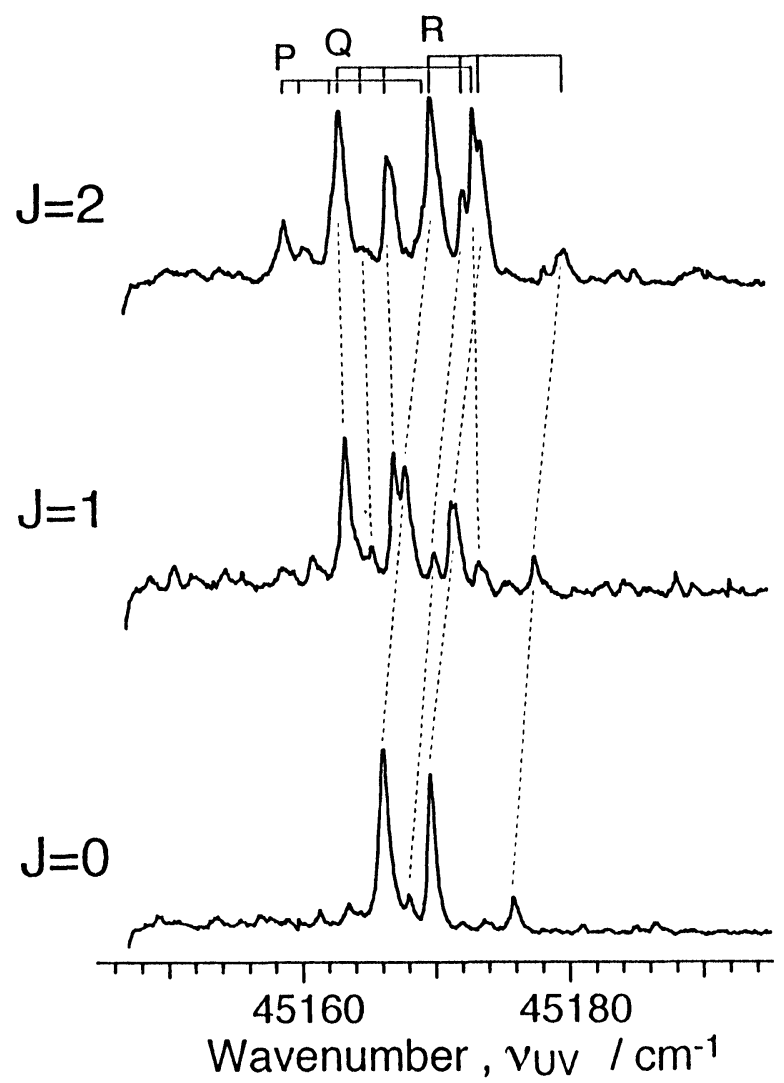

Figure 6 IR-UV double resonance MPI spectrum of acetylene in a supersonic jet around the band group $C$ obtained by exciting $\mathbf{J}^{\prime \prime}=0,1$, and 2 levels of the $v_{\mathrm{CH}}{ }^{\text {sym }}+v_{\mathrm{CH}}{ }^{\text {ant }}$ combination vibration by $v_{\mathrm{IR}}$. The horizontal scale was drawn by the frequency of $v_{\mathrm{UV}}$.

the $\widetilde{\mathrm{A}}$ state similar to $\mathrm{C}$, and the band structure can be well explained by the perpendicular transition with at least four vibronic bands. On the other hand, the broad band $\mathrm{A}^{\prime}$ shows a different $\mathrm{J}$ dependence. As can be seen in the figure, this band appears in the spectrum from $\mathrm{J}=2$ and 4 , but disappears from $\mathrm{J}=0$. This behavior means that $\mathrm{A}^{\prime}$ is the transition to the vibronic sublevel of $\mathrm{K}=2$ or 3 which has rotational levels of $\mathbf{J} \geq 2$ or 3 . Since the initial state of $v_{\mathrm{CH}}^{\text {sym }}+v_{\mathrm{CH}}^{\text {ant }}$ has $\mathrm{K}=0$, this transition has a selection rule of $\Delta \mathrm{K}=2$ or 3 . The $\Delta \mathrm{K}=2$ transition due to the deviation from the symmetric top has been found in the $\widetilde{A}-\widetilde{X}$ band system; however its intensity is very low. Thus the broad band $\mathrm{A}^{\prime}$ cannot be assigned to the vibronic band of $\widetilde{A}$. The natural interpretation is that the band $\mathrm{A}^{\prime}$ is the higher excited state in the region above the ionization potential and is excited by a non-resonant twophoton transition from $v_{\mathrm{CH}^{\mathrm{sym}}}+v_{\mathrm{CH}}^{\text {ant }}$ in the $\widetilde{\mathrm{X}}$ state. Since the intensity of band $\mathrm{A}^{\prime}$ shows quadratic dependence for the laser power of $\nu_{\mathrm{UV}}$, this higher excited state must be ionized by autoionization. The broad band width is consistent with the fast autoionization. However, such a non-resonant two-photon transition does not usually 


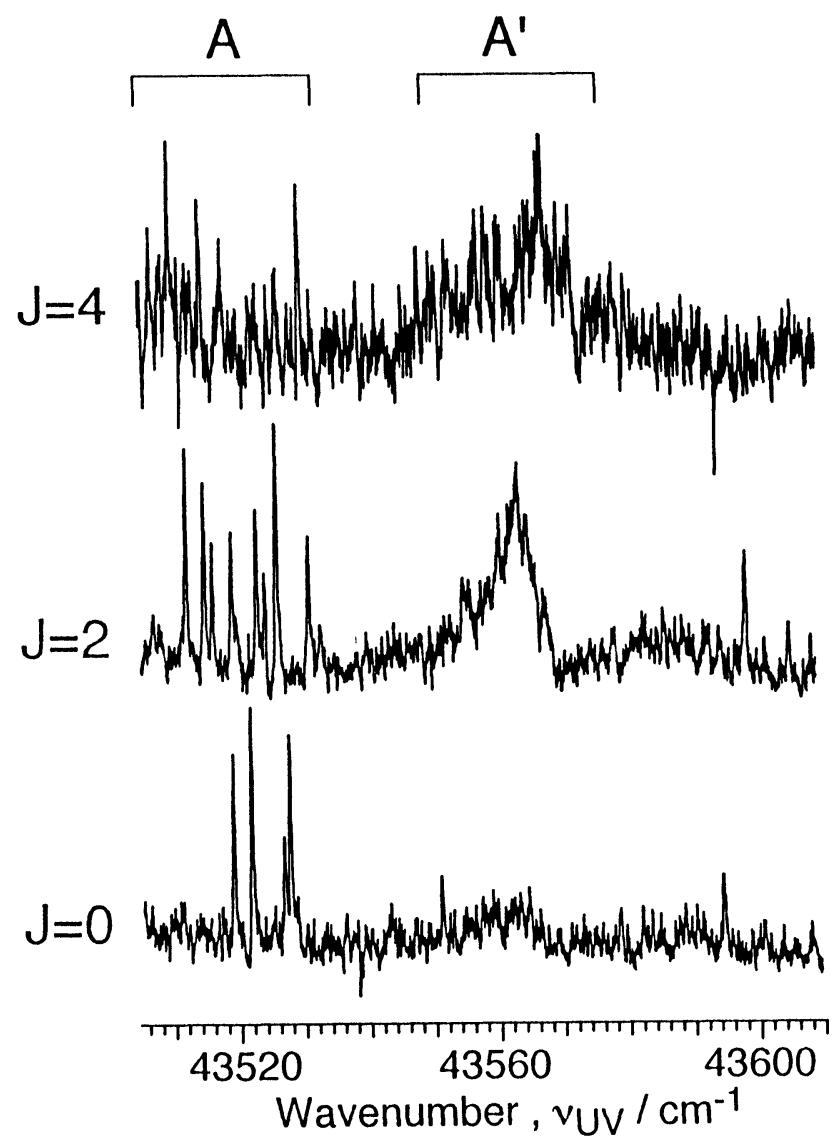

Figure 7 IR-UV double resonance MPI spectrum of acetylene in a supersonic jet around the band groups $\mathrm{A}$ and $\mathrm{A}^{\prime}$ obtained by exciting $\mathrm{J}^{\prime \prime}=0,2$, and 4 levels of the $v_{\mathrm{CH}}{ }^{\text {sym }}+v_{\mathrm{CH}}{ }^{\text {ant }}$ combination vibration by $v_{\mathrm{IR}}$. The horizontal scale was drawn by the frequency of $v_{\mathrm{UV}}$.

have much intensity. The transition intensity of band $\mathrm{A}^{\prime}$ may be enhanced by the near resonant effect to the sharp band system $\mathrm{A}$ at the first photon of $v_{\mathrm{Uv}}$.

The sharp band groups $\mathrm{A}, \mathrm{B}$ and $\mathrm{C}$ are assigned to the vibronic bands of the $\widetilde{\mathrm{A}}$ state, and their vibrational energies are about 7,865, 8,810, and $9,515 \mathrm{~cm}^{-1}$, respectively. Each group consists of several vibronic bands. This spectral feature shows that the vibronic levels in this region are strongly mixed with each other. It is straightforward to interpret it by Fermi resonance; each group has a main vibronic band which has a large absorption cross section from $\bar{X}$, and its intensity is distributed to nearby dark levels through Fermi resonance. At the present time, it is difficult to assign all the observed bands, therefore, we will discuss the assignments of the main band in each group.

The ordinary $\widetilde{\mathrm{A}} \leftarrow \mathbb{\mathrm { X }}$ absorption spectrum shows a long progression of the transbending vibration $\mathrm{nV}(\mathrm{n}=0,1,2, \ldots)$ and its intensity increases with an increase of the vibrational quantum number $\mathrm{n}$. The larger Franck-Condon factor in higher $\mathrm{n}$ 
is due to the geometrical change from linear to trans-bent structure. This intensity distribution for the progression of the mode $\mathrm{V}$ is also true in the transition from the $v_{\mathrm{CH}}^{\text {sym }}+v_{\mathrm{CH}}^{\text {ant }}$ vibrational level. In the region below the predissociation threshold, the first and the second members of the progression, $v_{\mathrm{CH}}^{\text {ant }}$ and $v_{\mathrm{CH}}{ }^{\text {ant }}+\mathrm{V}$, have already been found in the double resonance spectrum (see Figure 2). The band intensity largely increases in going from the first to the second member. Therefore it is possible to assign the main bands to the members of the progression $v_{\mathrm{CH}}{ }^{\text {ant }}+\mathrm{nV}$, where $\mathrm{n}=5,6$ and 7 for the main band of the groups $\mathrm{A}, \mathrm{B}$ and $\mathrm{C}$. The progression $\mathrm{nV}$ can start from the $v_{\mathrm{CH}}^{\text {sym }}+v_{\mathrm{CH}}^{\text {ant }}$ combination vibration in $\widetilde{\mathrm{A}}$, because the molecule is excited to the $v_{\mathrm{CH}}^{\text {sym }}+v_{\mathrm{CH}}^{\text {ant }}$ level in $\mathbb{X}$ by $v_{\mathrm{IR}}$. In these assignments, the main bands of $\mathrm{A}, \mathrm{B}$, and $\mathrm{C}$ correspond to $\mathrm{n}=2,3$ and 4 in the progression $v_{\mathrm{CH}}^{\text {sym }}+v_{\mathrm{CH}}^{\text {ant }}+\mathrm{nV}$. However this assignment has a disadvantage in that the main bands of $\mathrm{A}, \mathrm{B}$, and $\mathrm{C}$ consist of a lower overtone of the trans-bending vibration $\mathrm{V}$. The lower overtone of the mode $\mathrm{V}$ has a smaller Franck-Condon factor. Therefore we tentatively assigned the main bands of $\mathrm{A}, \mathrm{B}$ and $\mathrm{C}$ to be the progression, $v_{\mathrm{CH}}{ }^{\text {ant }}+$ $\mathrm{nV}(\mathrm{n}=5,6$, and 7$)$.

Let us check the validity of this assignment. The difference between $\mathrm{A}$ and $\mathrm{B}$ is $945 \mathrm{~cm}^{-1}$, which is a reasonable frequency of the trans-bending vibration in a higher overtone. ${ }^{31,32}$ From the tentative assignments $v_{\mathrm{CH}}{ }^{\text {ant }}+5 \mathrm{~V}$ for $\mathrm{A}$ and $v_{\mathrm{CH}}{ }^{\text {ant }}$ $+6 \mathrm{~V}$ for $\mathrm{B}$, the vibrational energy of a member in the progression is obtained to be $\left(1,049 \mathrm{n}-9.43 \mathrm{n}^{2}+2,857\right) \mathrm{cm}^{-1}$, where $2,857 \mathrm{~cm}^{-1}$ is the vibrational energy of $v_{\mathrm{CH}}$ ant. Since we cannot specify the main band in the group, the round numbers 7,865 and $8,810 \mathrm{~cm}^{-1}$ are used as the energy of A and B. In spite of the approximate values of the energies, this formula gives a reasonable value $1,039+2,857 \mathrm{~cm}^{-1}$ for the vibrational energy of the $v_{\mathrm{CH}}{ }^{\text {ant }}+\mathrm{V}$ level $(\mathrm{n}=1)$, which is measured to be $3,894=1,037+2,857 \mathrm{~cm}^{-1}$. This agreement supports the validity of these tentative assignments for $\mathrm{A}$ and $\mathrm{B}$.

In contrast, the assignment $v_{\mathrm{CH}}^{\text {ant }}+7 \mathrm{~V}$ may not be appropriate for the main band of $\mathrm{C}$. The difference between $\mathrm{B}$ and $\mathrm{C}$ corresponds to the frequency of the trans-bending vibration; however, the observed difference $705 \mathrm{~cm}^{-1}$ is too small to assign it to the trans-bending vibration. In actuality, the above formula gives the vibrational energy of the $v_{\mathrm{CH}}^{\text {ant }}+7 \mathrm{~V}$ level to be $9,736 \mathrm{~cm}^{-1}$ (vibronic energy $\left.51,934 \mathrm{~cm}^{-1}\right)$ which is about $200 \mathrm{~cm}^{-1}$ larger than the observed vibrational energy of $C\left(9,515 \mathrm{~cm}^{-1}\right)$. If we insist on this assignment, an anharmonicity in higher order or a strong level interaction must be introduced. Thus it may be suggested that the main band of $\mathrm{C}$ should be assigned to another combination vibration, such as $v_{\mathrm{CH}}^{\text {sym }}$ $+v_{\mathrm{CH}}^{\text {ant }}+v_{\mathrm{CC}}+2 \mathrm{~V}$, of which the calculated energy is $9,383 \mathrm{~cm}^{-1}$ without considering anharmonicity.

Whether the main band of $\mathrm{C}$ is assigned to $v_{\mathrm{CH}}{ }^{\text {ant }}+7 \mathrm{~V}$ or not, the higher member of the progression is found to disappear. As can be seen in the figure, no band has been found in the region above the band group C. It is highly probable that the progression of the trans-bending vibration appears in the $\widetilde{\mathrm{A}} \leftarrow \overline{\mathrm{X}}$ transition of acetylene. Therefore, if the main band of $\mathrm{C}$ is not assigned to $v_{\mathrm{CH}}{ }^{\text {ant }}+7 \mathrm{~V}$, the 
disappearance of $v_{\mathrm{CH}}^{\text {ant }}+7 \mathrm{~V}$ must be explained. If the main band of $\mathrm{C}$ can be assigned to $v_{\mathrm{CH}}^{\text {ant }}+7 \mathrm{~V}$, the next member of the progression $v_{\mathrm{CH}}{ }^{\text {ant }}+8 \mathrm{~V}$ must be observed because the spectrum covers more than $1,000 \mathrm{~cm}^{-1}$ region above $\mathrm{C}$. Therefore the disappearance of the higher member of the progression $v_{\mathrm{CH}}{ }^{\text {ant }}+\mathrm{nV}$ is derived by both possible assignments of $\mathrm{C}$.

It is appropriate to explain the disappearance of the vibronic band by the decrease in ionization efficiency. The decrease in the fluorescence quantum yield is interpreted as an increase in the nonradiative rate. Similarly, the efficiency of the multiphoton ionization process decreases if the resonant level has a relaxation process whose rate is comparable to or larger than the ionization rate. Therefore the disappearance of the vibronic band suggests that the nonradiative relaxation is accelerated much in the region above the band group C, i.e. $\geq 9,546 \mathrm{~cm}^{-1}\left(51,744 \mathrm{~cm}^{-1}\right.$ in the total energy). Since the ionization signal is observed in the region above the predissociation threshold, the ionization rate in the present condition is much larger than the predissociation. Thus the acceleration of the nonradiative process may be due to the opening of another relaxation channel. The relaxation mechanism will be revealed by the detailed analysis of the IR-UV double resonance spectrum.

In conclusion, IR-UV double resonance spectroscopy has been applied to the $\widetilde{A}-\bar{X}$ transition of acetylene in gas and in a supersonic jet. The electronic transition of the $v_{\mathrm{CH}}^{\text {ant }}$ and the $v_{\mathrm{CH}}^{\text {sym }}+v_{\mathrm{CH}}^{\text {ant }}$ vibrationally excited molecule has been observed. In the region below the predissociation threshold, the $v_{\mathrm{CH}}^{\text {ant }}$ vibration in $\widetilde{\AA}$ is strongly observed when the $v_{\mathrm{CH}}{ }^{\text {sym }}+v_{\mathrm{CH}}{ }^{\text {ant }}$ combination vibration in $\mathbb{X}$ is excited by the IR laser $v_{\mathrm{IR}}$. In contrast, the $v_{\mathrm{CH}}^{\text {ant }}$ vibration appears very weak when the $v_{\mathrm{CH}}{ }^{\text {ant }}$ fundamental vibration is excited by $\nu_{\mathrm{IR}}$, and the in-plane cis-bending vibration $v_{\mathrm{cis}}(\mathrm{in})$ appears strongly. The difference has been explained by the strong Fermi resonance of the $v_{\mathrm{CH}}{ }^{\text {ant }}$ fundamental vibration in $\bar{X}$. The vibrational frequency of $v_{\mathrm{CH}}{ }^{\text {ant }}$ and $v_{\text {cis }}\left(\right.$ in) in the $\widetilde{A}$ state has been measured to be 2,857 and $785 \mathrm{~cm}^{-1}$, respectively. From the decrease in the fluorescence quantum yield, the predissociation threshold was newly determined to be $46,439 \sim 46,673 \mathrm{~cm}^{-1}(133.11 \pm 0.33 \mathrm{kcal} / \mathrm{mol})$. In the region above the predissociation threshold, three clusters of the vibronic bands are found, and the vibrational mixing due to Fermi resonance in $\widetilde{A}$ has been suggested. The main bands of three clusters are assigned to the progression of the trans-bending vibration starting from $v_{\mathrm{CH}}{ }^{\text {ant }}$. From the disappearance of the higher members of the progression, the acceleration of the nonradiative relaxation in the region above $51,744 \mathrm{~cm}^{-1}$ is suggested.

\section{Acknowledgment}

We thank Professor F. F. Crim (University of Wisconsin) and Professor M. Herman (Université Libre de Bruxelles) for showing us their papers prior to publication. We also thank Dr. M. Bautista and Mr. K. Takazawa of Waseda University for their kind revision of the manuscript. 


\section{References}

1. J. Pliva, P. Escherick and A. Owyoung. J. Mol. Spctrosc., 125, 393 (1987).

2. V. A. Venturo, P. M. Maxton and P. M. Felker. J. Phys. Chem., 96, 5234 (1992).

3. V. A. Venturo, P. M. Maxton and P. M. Felker. Chem. Phys. Lett., 198, 628 (1992).

4. B. F. Henson, G. V. Hertland, V. A. Venturo and P. M. Felker. J. Chem. Phys., 97, 2189 (1992).

5. V. A. Venturo, P. M. Maxton, B. F. Henson and P. M. Felker. J. Chem. Phys., 96, 7855 (1992).

6. T. Ebata, M. Hamakado, S. Moriyama, Y. Morioka and M. Ito. Chem. Phys. Lett., 199, 33 (1992).

7. M. Takahashi, Y. Okuzawa, M. Fujii and M. Ito. Can. J. Chem., 69, 1656 (1991).

8. R. H. Page, Y. R. Shen and Y. T Lee. J. Chem. Phys., 88, 5362 (1988).

9. R. H. Page, Y. R. Shen and Y. T Lee. J. Chem. Phys., 88, 4621 (1988).

10. M. J. Frost and I. W. M. Smith. Chem. Phys. Lett., 191, 574 (1992).

11. T. M. Ticich, M. D. Likar, H. R. Dübal, L. J. Butler and F. F Crim. J. Chem. Phys., 87, 5820 (1987).

12. A. Shinha, R. L. Vander Wal, L. J. Butler and F. F. Crim. J. Phys. Chem., 91, 4645 (1987).

13. M. D. Likar, J. E. Baggot, A. Shinha, T. M. Ticich, R. L. Vander Wal and F. F. Crim. J. Chem. Soc. Faraday Trans. 2, 84, 1483 (1988).

14. M. D. Likar, A. Shinha, T. M. Ticich, R. L. Vander Wal, and F. F. Crim. Ber. Bunsenges. Phys. Chem., 92, 289 (1988).

15. M. D. Likar, J. E. Baigot and F. F. Crim. J. Chem. Phys., 90, 6266 (1989).

16. A. Shinha, R. L. Vander Wal and F. F. Crim. J. Chem. Phys., 91, 2929 (1989).

17. A. Shinha, R. L. Vander Wal and F. F. Crim. J. Chem. Phys., 92, 401 (1990).

18. R. L. Vander Wal and F. F. Crim. J. Phys. Chem., 93, 5331 (1989).

19. R. L. Vander Wal, J. L. Scott and F. F. Crim. J. Chem. Phys., 92, 803 (1990).

20. M. Fujii, A. Haijima and M. Ito. Chem. Phys. Lett., 150, 380 (1988); A. Haijima, M. Fujii and M. Ito. J. Chem. Phys., 92, 959 (1990).

21. A. L. Utz, J. D. Tobiason, E. Carrasquillo M., J. L. Sanders and F. F. Crim. J. Chem. Phys., 98, $2742(1993)$.

22. J. D. Tobiason, A. L. Utz and F. F. Crim. J. Chem. Phys., 99, 928 (1993).

23. Y. Okuzawa, M. Fujii and M. Ito. Chem. Phys. Lett., 171, 341 (1990).

24. G. Herzberg, Molecular Spectra and Molecular Structure III. Electronic Spectra and Electronic Structure of Polyatomic Molecules (Van Nostrand Reinhold, New York, 1966).

25. G. Herzberg, Molecular Spectra and Molecular Structure II. Infrared and Raman Spectra of Polyatomic Molecules (Van Nostrand Reinhold, New York, 1966).

26. J. C. Van Craen, M. Herman, R. Colin and J. K. G. Watson. J. Mol. Spectrosc., 111, 185 (1985).

27. J. K. G. Watson, M. Herman, J. C. Van Craen and R. Colin. J. Mol. Spectrosc., 95, 101 (1982).

28. B. C. Smith and J. S. Winn. J. Chem. Phys., 89, 4638 (1988).

29. A. Baldacci, S. Ghersetti and K. N. Rao. J. Mol. Spectrosc., 68, 183 (1977).

30. M. Peric, S. D. Peyerimhoff and R. J. Buenker. Mol. Phys., 62, 1339 (1987).

31. J. C. Van Craen, M. Herman, R. Colin and J. K. G. Watson. J. Mol. Spectrosc., 119, 137 (1986).

32. D. D. Foo and K. K. Innes. Chem. Phys. Lett., 22, 439 (1973) 